\title{
Turbulence characteristics of supersonic corner flows in a low aspect ratio rectangular channel
}

\author{
Rohan R. Morajkar*, Mirko Gamba ${ }^{\dagger}$ \\ Department of Aerospace Engineering, University of Michigan, Ann Arbor, MI 48105
}

\begin{abstract}
In this study we present the experimental results of stereo particle image velocimetry conducted in the corner of a Mach 2.75 low aspect ratio rectangular channel flow. Data is recorded at multiple cross-section planes and analyzed to study the formation of corner vortices and their effects on the structure of the mean flow field. Results show that several vortex elements exist instantaneously in the corner, however they do not conform to the time-averaged secondary flow structure proposed by previous studies. Decomposing the flow into a large and small scale components, along with conditional averaging, makes it possible to capture and describe the properties of, the large scale corner vortices which are similar to the description of corner vortex pair proposed by previous studies. A conditional definition of corner vortex pair in instantaneous fields is introduced which facilitates the statistical study of their properties.
\end{abstract}

\section{Nomenclature}

$\begin{array}{lll}x & =\text { Streamwise flow direction } \\ y & =\text { Side-wall normal direction } \\ z & =\text { Bottom-wall normal direction } \\ U & =\text { Ensemble-average streamwise flow velocity component } \\ V & =\text { Ensemble-average flow velocity in } y \text {-direction } \\ W & =\text { Ensemble-average flow velocity in } z \text {-direction } \\ u & =\text { Instantaneous velocity in } x \text {-direction } \\ v & =\text { Instantaneous velocity in } y \text {-direction } \\ w & =\text { Instantaneous velocity in } z \text {-direction } \\ t & =\text { Wall tangential direction } \\ n & =\text { Wall normal direction } \\ \delta_{99} & =\text { P9 } \% \text { boundary layer thickness } \\ \omega_{R R} & =\text { Conventional vorticity } \\ \omega & =\text { Derivative in respective direction } \\ \text { Subscripts: } & =\text { Quantity evaluated at the wall } \\ x, y, z & =\text { Luantity evaluated in freestream } \\ w & =\text { Eluctuation quantity } \\ \infty & =\end{array}$

*Graduate Student Research Assistant, AIAA Member, rohanrm@umich . edu

${ }^{\dagger}$ Assistant Professor, AIAA Member, mirkog@umich . edu 


\section{Introduction}

Flows along a streamwise corner are quite common in aerospace applications. Such flows are commonly found along the wing-body junction of an aircraft and rectangular air intake ducts of aircrafts. These flows often give rise to secondary transverse flow perpendicular to the streamwise direction of the bulk flow. Secondary flows play a role in convection of mass, momentum, vorticity and energy from the primary flow into the corner and from the corner into the primary flow [1]. Due to this, the secondary flows often influence the structure of the primary and thus deviate from a canonical structure. This may reduce the effective area available for the flow inside the duct thereby affecting the performance of the aircraft or induce external separations along the wing-body junction causing loss of lift. Corner flows are characterized by regions of low momentum fluid near the junction of the walls forming the corner. These regions are particularly susceptible to separation especially in sensitive flows involving complex phenomena like shock-boundary layer interactions. It is thus required to understand the characteristics of such a flow. The aim of this work is to study supersonic turbulent corner flows that would exist in the air intake of a supersonic aircraft and attempt to understand the structure and the driving properties of the flow. As is evident from previous research on shock-boundary layer interactions [2,3], strong incipient separation is fairly a common place in such type of flows. These incipient separation bubbles are primarily caused by the interaction of spatially unsteady structures which are a result of unsteady flowfields.

Previous studies on the structure of corner flows $[4,5]$ have primarily used intrusive techniques such as Preston/pitot tubes and hot wire in order to study the secondary flows in the corners of ducted supersonic flows. They have established that secondary flows comprise, on a mean sense, of two counter rotating vortices forcing the fluid towards the corner. These previous works have attempted to explain the source of these vortices as the result of anisotropic gradients in the Reynolds stress that exist as the corner is approached. However, the use of these intrusive techniques may substantially modify the flow in low aspect ratio rectangular channels, and thus present a mean picture of the flow field that might be a distorted representation of the instantaneous flow phenomena taking place in the corner. Thus, there is a need for non-intrusive measurements to study the evolution of corner vortices in such flows.

This study primarily attempts to understand the instantaneous turbulent flow field formed in the corner of a supersonic (Mach 2.75) duct flow which would be ubiquitous in supersonic rectangular air intake systems using particle image velocimetry measurements. The measurements specifically focus on the turbulent structure of the boundary layers developing along orthogonal walls as they merge in the corner.

\section{Previous Research on Supersonic Corner Flows}

The fluid flow which exists at the intersection of two walls is characterized by secondary motion, which are defined as flow motions due to velocity components normal to the principal flow direction. Ridha [6] published a review article describing many of the theoretical studies of the corner flow boundary layer that have been conducted. Rubin [7] initially developed an analytical model to describe the laminar corner flow. He divided the flow cross-section into 4 parts: boundary layers along the two walls forming the corner, the corner overlap between the boundary layers and the core flow. The terms of the Navier-Stokes (N-S) and boundary layer equations were then expanded into an infinite series and the constants matched up to second degree in order to match the values of various components of velocity that asymptote towards the core flow and in the boundary layer overlap region. He thus obtained a numerical solution describing the flow in corner region.

Prandtl in 1952 formally divided the secondary flows into two categories: secondary flows of the first and second kinds, which have been further explained by Bradshaw [8]. Secondary flows of the first kind originate because of mean flow skewing (i.e., due to the tilting and stretching term in the vorticity equation), and are relatively well understood. However, much is to be known about secondary flows of the second kind, which are a result of anisotropic variations of Reynolds stresses [1, 6]. The streamwise corner flows which exist at the intersection of two surfaces intersecting in the principal flow direction fall into the second kind.

It is believed that secondary corner flows are characterized by streamwise vortices that arise due to the production of vorticity by Reynolds stresses. The pair of streamwise vortices that would exist in ideal symmetric flow conditions 
is shown in Figure 1. Perkins [9] explains the formation of these vorticies from the (steady) $x$-vorticity conservation equation written after the Reynolds decomposition and averaging is performed; thus taking into account the Reynolds stresses, the vorticity equation can be written as:

$$
U \frac{\partial \omega_{x}}{\partial x}+V \frac{\partial \omega_{x}}{\partial y}+W \frac{\partial \omega_{x}}{\partial z}=\nu \nabla^{2} \omega_{x}+\omega_{x} \frac{\partial U}{\partial x}+P 1+P 2+P 3+P 4
$$

where the terms on the left-hand-side represent the convection of the streamwise component of vorticity $\left(\omega_{x}\right)$. The first two terms on the right-hand-side are the viscous diffusion and vortex stretching, respectively. The third term is

$$
P 1=\omega_{y} \frac{\partial U}{\partial y}+\omega_{z} \frac{\partial U}{\partial z}
$$

and describes the production of streamwise vorticity by skewing of the mean flow field and thus represents the secondary flow of the first kind. The fourth terms is

$$
P 2=\frac{\partial}{\partial x}\left(\frac{\partial \overline{u^{\prime} v^{\prime}}}{\partial z}-\frac{\partial \overline{u^{\prime} w^{\prime}}}{\partial y}\right)
$$

and describes the formation of vorticity due to streamwise gradients of the primary Reynolds shear stress gradients. This term is often ignored from most of the studies due to assumption of zero pressure gradients; whether this term is important in flows with strong pressure gradients (e.g., in locations where a shock wave induced pressure gradient is present) is currently not known. The fourth term in the vorticity equation is

$$
P 3=\frac{\partial^{2}}{\partial y \partial z}\left(\overline{v^{\prime 2}}-\overline{w^{\prime 2}}\right)
$$

and represents the formation of vorticity due to anisotropy in direct turbulent stresses. This term is believed to be the prominent quantity that leads to creation of streamwise vortices in a corner. Unlike the isotropic nature of the turbulence in the undisturbed boundary layers away from the corner, the kinetic energy is distributed unequally along different directions in the corner region, which leads $P 3$ to take non-zero values. The final term in the vorticity equation is

$$
P 4=\left(\frac{\partial^{2}}{\partial z^{2}}-\frac{\partial^{2}}{\partial y^{2}}\right) \overline{v^{\prime} w^{\prime}}
$$

and depicts the formation of streamwise vorticity due to gradients in the secondary stress $\overline{v^{\prime} w^{\prime}}$. Together $P 2, P 3$ and $P 4$ represent the quantities responsible for the development and sustenance of the secondary flow of the second kind. These terms are important in corner flows.

Gessner [1] experimentally evaluated the terms in the energy and vorticity balance equations using pressure taps and hot wire measurements. His results suggest that the transverse secondary flow is initiated as a direct result of the primary turbulent stress gradients normal to the corner bisector and that the anisotropy of the stresses does not play a major role in the secondary flow generation as suggested by Perkins[9]. He suggests that the variations in the curvature of iso-contours of the axial velocity that occur in the flow undergoing laminar-to-turbulent transition result into turbulent stress gradients along the iso-contour which necessitate the development of a secondary flow from the concave to the convex side of the iso-contour. Brundrett and Baines [10] conducted experimental investigation using hot-wire measurements in order to characterize regions of vorticity creation and diffusion. Their results suggest that the diffusion of vorticity peaks near the wall while the production of vorticity is associated with the region near the corner bisector; however, it is zero on the corner bisector itself while being of opposing signs on either side of it. They suggest that it is because of this distribution that the vortices have a tendency to convect towards the walls. The zone of peak vorticity production is characterized by a vortex on each side of the corner bisector with opposite signs. It was found that the vorticity production is independent of the Reynolds number, but the vortex diffusion is not. They suggested this to be the reason for the secondary flow to be pushed towards the wall as the Reynolds number is increased. Kornilov and Kharitonov [11] studied the structure of the corner flows in asymmetric configurations 
featuring unequal boundary layer thicknesses on the walls forming corners. One of their most important findings was that the vortex located towards the thicker boundary layer is stronger and larger than that towards the thinner boundary layer.

The unstable nature of these flows have been reported by various stability studies conducted by Alizard et al. [12], Zamir [13] and Balachandar and Malik [14]. As a result of this unstable nature it is highly likely that any intrusive form of measurement would lead to a disturbed flow, thus yielding false measurements of the corner flow properties, especially in supersonic flow wherein the effect of shock wave / flow interaction resulting from the probe can propagate upstream through the viscous region and change the characteristics of the incoming flow as it has been explained by Chapman et al. [15] and reported by Kornilov [16]. Because of this, it is necessary to characterize the flow using non-invasive experimental techniques, such as the particle image velocimetry (PIV) technique. Motivated by this observation, Park et al.[17] conducted PIV measurements in the corner flow in a plane oriented along the corner bisector in the streamwise direction. They majorly focused on capturing the developing boundary layer on this plane. Their findings are in agreement with the theoretical studies in that the velocity distributions were found to follow self-similar profiles and that the corner velocity profiles correspond to the Blasius branch of the theoretical solutions. However in order to fully understand the secondary flows, it is necessary to obtain non-intrusive high resolution measurements in the plane where such secondary flows are observed. Uruba et al. [18] conducted POD analyses to elucidate the most energetic modes of secondary flows in the rectangular corner.

This work builds on these earlier efforts, and aims to provide additional insights into the behavior of vorticies in the corner region. Additionally, this study proposes a systematic way to define the corner vortex pair in instantaneous turbulent vector fields where the identification of the vortex pair is masked by a forest of small-scale vortices associated with the underlying turbulent flow of the boundary layer.Thus, this paper will majorly be focused on analyzing the instantaneous structure of the corner flow from a set of instantaneous PIV measurements that focus into the turbulent boundary layers merging at the corner.

\section{Experimental Setup}

\section{A. Wind Tunnel setup}

The experiments presented here were carried out at the Michigan Glass Wind Tunnel (GWT) facility. A schematic diagram of the configuration used in the study is shown in Figure 2. It is a low aspect ratio suction supersonic wind tunnel $57.2 \mathrm{~mm} \times 69.3 \mathrm{~mm}(2.25 \mathrm{in} \times 2.75 \mathrm{in})$ in cross-section currently configured to operate nominally at Mach 2.75 with stagnation pressure and temperature of $98.1 \mathrm{kPa}$ and $294 \mathrm{~K}$, respectively. The effective (measured) Mach number is approximately 2.72. The tunnel is composed of a one-sided two-dimensional converging-diverging nozzle followed by a constant area test section. This design was selected to produce an equilibrium flat plate boundary layer [19] in an attempt to minimize pressure gradients history effects and Görtler vortices on the boundary layer developing on the bottom-wall (floor) of the wind tunnel. Since our previous work has focused on a 3D SBLI configuration and this work is an effort to better understand it, we use the same coordinate system of our previous SBLI work [3, 20, 21, 22]. In particular, the origin of the coordinate system is centered at the location of the leading edge of the full-span $6^{\circ}$ shock generator wedge (which is about $481.5 \mathrm{~mm}$ downstream of the nozzle throat) used in our previous work on 3D SBLI. However, in this work the shock generator has been removed. The unit Reynolds number of the flow is $8.9 \times 10^{6} / \mathrm{m}$ with an incoming boundary layer thickness $\left(\delta_{99}\right)$ of $10 \mathrm{~mm}$ measured at $x=75.5 \mathrm{~mm}$ and $y=28.6 \mathrm{~mm}$ (i.e., on the vertical midplane of the test section) in an empty tunnel [19]. The pressure gradient parameter defined as $\beta=\frac{\delta}{\left(\rho U^{2}\right)} \frac{\partial P}{\partial x}$ was calculated from side-wall static pressure measurements conducted in the empty tunnel at half the tunnel height. The value of this parameter was found to be $5 \times 10^{-4}$ for this experiment. A complete summary of the experimental conditions along with their respective uncertainties is provided in Table 1. Optical access to the test section is provided from both sides of the wind tunnel by glass windows that run along the whole length of the wind tunnel, including the nozzle throat region. 


\section{B. Stereo Particle Image Velocimetry (SPIV)}

SPIV measurements were performed. Two interline transfer CCD cameras (LaVision Flowmaster) recording at $3.33 \mathrm{~Hz}$ with a resolution of $1280 \times 1024$ pixel were used for the imaging. The cameras were placed in a stereoscopic, forwardscattering configuration oriented at $33^{\circ}$ with respect to the measurement plane. The cameras feature a minimum interframe time delay of about $500 \mathrm{~ns}$. The double-pulse illumination of the flow is provided by a pair of low repetition rate, frequency-doubled Nd:YAG lasers producing an output of $532 \mathrm{~nm}$ beam with a total energy of $200 \mathrm{~mJ} / \mathrm{pulse}$. The lasers are triggered at $10 \mathrm{~Hz}$ with a time delay of $650 \mathrm{~ns}$ in between the two pulses and pulse duration of $10 \mathrm{~ns}$. The delay is measured with a ThorLabs DT10A/M photodiode that has a $1 \mathrm{~ns}$ response time and a LeCroy Waverunner $6030350 \mathrm{MHz}$ digital oscilloscope and then ensured by adjusting the time delay between the trigger signals. In order to optimize the particle dropout and volumetric effects, laser sheet thickness is set using expanding-collimating optics to approximately 4 times the out of plane displacement of a particle within the measurement domain as suggested by Adrian and Westerweel [23]. The beam width was measured to be $1.45 \pm 0.25 \mathrm{~mm}$ which is approximately 3.6 times the particle displacement (approx. $400 \mu \mathrm{m}$ ) in $650 \mathrm{~ns}$ at the free-stream speed.

Particle seeding of the flow was generated by a TDA-4B portable Laskin nozzle aerosol. The generator consists of an array of six Laskin nozzles that create poly-dispersed sub-micron particles using Poly-Alpha Olefin (PAO) oil with density of $819 \mathrm{~kg} / \mathrm{m}^{3}$. The LaVision DaVis 8 software is used for the acquisition of the measurement and processing of the data. The three-component velocity fields are reduced from the particle images using Davis 8. A multi-pass with reducing interrogation window size is used. Two passes were conducted using an interrogation window size of $64 \times 64$ pixels with a 50\% overlap. The final size of the interrogation windows after two further passes was $32 \times 32$ pixels with an overlap of $75 \%$, which corresponds to a projected physical size of about $0.47 \mathrm{~mm} \times 0.47 \mathrm{~mm}$ and a vector spacing of about $0.15 \mathrm{~mm} \times 0.15 \mathrm{~mm}$ spanning a physical region of $17 \mathrm{~mm} \times 17 \mathrm{~mm}$. Post processing within multiple passes included deleting a vector if its correlation value was less than 0.8 as well as removing groups with less than 4 vectors. Post processing was also conducted once all the passes were completed by removing vectors with a peak ratio (Q) less than 1.2. Laser sheets were oriented perpendicular to the flow, spanning a fraction the cross-section of the tunnel. Multiple such images (about 1000 instances at each measurement location) were recorded to construct statistics of various flow properties.

The data (each snapshot/instantaneous field) was further validated in Matlab using the criterion proposed by Nogueira et al. [24]. The missing data was then interpolated using a fourth order differential equation for interpolating data [25]. The method leaves all known values intact. The data was lowpass filtered using a Gaussian filter with a standard deviation corresponding to 2.5 vector spacing and a kernel size of $5 \times 5$ points, which in physical space corresponds to size of $0.75 \mathrm{~mm} \times 0.75 \mathrm{~mm}$. All of the SPIV data was analyzed using PIVMAT [26] modified to use a least-square finite difference scheme for computing derivatives except at the edges where forward and/or backward differencing was used.

Measurements on transverse vertical (TV) planes are performed on the lower right corner (as one looks downstream) of the duct cross-section at three streamwise direction: (TV1) $-100 \mathrm{~mm}$, (TV2) $-50 \mathrm{~mm}$ and (TV3) $75 \mathrm{~mm}$ with respect to the location where we have positioned the leading edge of a shock generator in our previous work [27,3].

\section{Flow field scale decomposition and definition of corner flow properties}

Before we present the results of this study we provide a few initial definitions of quantities to be used in the analysis section that follows. These quantities are introduced to better capture the corner flow structure. In particular, to more effectively describe the instantaneous flowfield, it is decomposed into two components separating the "large" and "small" scale contribution to the structure of the corner vortex. Then, the average corner flow structure is reconstructed from conditionally interrogating the decomposed flowfield.

\section{A. Flow field scale decomposition}

The instantaneous three-component velocity field is decomposed using two decompositions of motion. First, a scaledependent decomposition of motion into a large and small scale contribution is applied. Then, the resulting flow fields 
are additionally decomposed into a fluctuating and ensemble-average components using the Reynolds decomposition of motion. In this latter decomposition, we assume that the flow is not far from behaving like an incompressible flow within the boundary layer, $|\partial p / \partial x|<<1$ and that the flow is statistically stationary.

Let $\mathbf{u}=u \hat{\mathbf{i}}+v \hat{\mathbf{j}}+w \hat{\mathbf{k}}$ be the three-component velocity field in the domain. The flow can be decomposed into a large-scale and a small-scale contribution as

$$
\mathbf{u}=\mathbf{u}_{L}+\mathbf{u}_{S}
$$

where we refer to $\mathbf{u}_{L}$ as the velocity field of the large scales and $\mathbf{u}_{S}$ is the velocity field of the small scales. More specifically, the large/small scale decomposition is conducted by defining a (filtered) velocity field over a scale $\Omega$ :

$$
\mathbf{u}_{L}(\mathbf{x}, t)=\frac{\iiint_{\Omega} h(\mathbf{s}) \mathbf{u}(\mathbf{x}+\mathbf{s}, t) \mathrm{d} \mathbf{s}}{\iiint_{\Omega} h(\mathbf{s}) \mathrm{d} \mathbf{s}}
$$

where $h(\mathbf{s})$ is an appropriate low pass filtering kernel function (e.g., a top-hat filer). In this paper the filtering kernel function is a top hat filter spanning a square of $25 \times 25$ data nodes or approximately $0.5 \delta \times 0.5 \delta$ locally. A discussion on the choice of this filtering kernel size and its implications for what presented in this work is given subsequently. The small scale contribution is then defined as:

$$
\mathbf{u}_{S}(\mathbf{x}, t)=\mathbf{u}(\mathbf{x}, t)-\mathbf{u}_{L}(\mathbf{x}, t)
$$

This decomposition can be extended to average fields due to the linearity of the averaging and field decomposition processes. Additionally, we invoke the Reynolds decomposition where the velocity field is decomposed as the sum of an average field and an instantaneous fluctuation component

$$
\mathbf{u}=\mathbf{U}+\mathbf{u}^{\prime}
$$

Finally, we can combine the two decompositions due to the linearity of each step. Thus we can write

$$
\mathbf{u}=\left(\mathbf{U}_{L}+\mathbf{u}_{L}^{\prime}\right)+\left(\mathbf{U}_{S}+\mathbf{u}_{S}^{\prime}\right)
$$

Substituting in the continuity equation, under the incompressible assumption, we have

$$
\nabla \cdot\left(\mathbf{u}_{L}+\mathbf{u}_{S}\right)=0
$$

from which it also follows

$$
\nabla \cdot\left(\mathbf{U}_{L}+\mathbf{U}_{S}\right)=0
$$

and thus

$$
\nabla \cdot\left(\mathbf{u}_{L}^{\prime}+\mathbf{u}_{S}^{\prime}\right)=0
$$

We then apply this decomposition of motion to the momentum equation, from which we can derive a corresponding form of the three-component vorticity equation in terms of the propose decomposition of scales. In index notation, the resulting (approximate) vorticity equation can be written as:

$$
U_{i} \frac{\partial}{\partial x_{i}} \overline{\omega_{j}}=\overline{\omega_{i}} \frac{\partial}{\partial x_{i}} U_{j}+\frac{\partial}{\partial x_{i}}\left(\overline{\omega_{i}^{\prime} u_{j}^{\prime}}-\overline{\omega_{j}^{\prime} u_{i}^{\prime}}\right)+\nu \frac{\partial^{2}}{\partial x_{i}^{2}} \overline{\omega_{j}}
$$

In particular, because in this study we are primarily interested in analyzing vortical features organized along the streamwise direction, the corresponding $x$-component of the vorticity equation 14 becomes:

$$
\begin{array}{r}
{\left[U \frac{\partial \overline{\omega_{x}}}{\partial x}+V \frac{\partial \overline{\omega_{x}}}{\partial y}+W \frac{\partial \overline{\omega_{x}}}{\partial z}\right]=\left[\overline{\omega_{x}} \frac{\partial U}{\partial x}+\overline{\omega_{y}} \frac{\partial U}{\partial y}+\overline{\omega_{z}} \frac{\partial U}{\partial z}\right]+\left[\frac{\partial}{\partial y}\left(\overline{\omega_{y}^{\prime} u^{\prime}}-\overline{\omega_{x}^{\prime} v^{\prime}}\right)+\frac{\partial}{\partial z}\left(\overline{\omega_{z}^{\prime} u^{\prime}}-\overline{\omega_{x}^{\prime} \omega^{\prime}}\right)\right]+} \\
+\nu\left(\frac{\partial^{2} \overline{\omega_{x}}}{\partial x^{2}}+\frac{\partial^{2} \overline{\omega_{x}}}{\partial y^{2}}+\frac{\partial^{2} \overline{\omega_{x}}}{\partial z^{2}}\right)
\end{array}
$$


where the different terms of equation 15 can be recognized and grouped into $P 1, P 2, P 3$ and $P 4$ defined previously in equation 1.

The vortex tilting term is

$$
P 1=\overline{\omega_{y}} \frac{\partial U}{\partial y}+\bar{\omega}_{z} \frac{\partial U}{\partial z}
$$

For the case of small adverse pressure gradient $(|\beta|<<1)$, it can be approximated by noting that

$$
|\partial V / \partial x| \&|\partial W / \partial x|<<|\partial U / \partial y| \&|\partial U / \partial z|
$$

The approximations are justified by invoking the boundary layer approximation and the observation from our measurements that the streamwise changes in $\bar{v}$ and $\bar{w}$ across different streamwise measurement locations remains small even as the corner is approached [28]. One implication of this approximation is that the vorticity components in a plane perpendicular to the flow direction can be approximated as

$$
\overline{\omega_{y}} \simeq \frac{\partial U}{\partial z}
$$

and

$$
\overline{\omega_{z}} \simeq-\frac{\partial U}{\partial y}
$$

which would implies that $P 1 \simeq 0$, i.e., the mean vorticity and shear would not contribute to the reorganization of existing vorticity into the streamwise direction to form the corner vortex pair.

The different terms of the approximate equation 15 , which is still written in terms of the ensemble-average quantities, can then be decomposed using the scale decomposition proposed above. Thus, the following form of the terms on the right-hand-side of equation 15 can be obtained:

$$
\begin{gathered}
P 1=\left(\overline{\omega_{L_{y}}}+\overline{\omega_{S_{y}}}\right) \frac{\partial\left(U_{L}+U_{S}\right)}{\partial y}+\left(\overline{\omega_{L_{z}}}+\overline{\omega_{S_{z}}}\right) \frac{\partial\left(U_{L}+U_{S}\right)}{\partial z} \\
P 2=\frac{\partial}{\partial x}\left[\frac{\partial\left[\overline{\left(u_{L}^{\prime}+u_{S}^{\prime}\right)\left(v_{L}^{\prime}+v_{S}^{\prime}\right)}\right]}{\partial z}-\frac{\partial\left[\overline{\left(u_{L}^{\prime}+u_{S}^{\prime}\right)\left(w_{L}^{\prime}+w_{S}^{\prime}\right)}\right]}{\partial y}\right] \\
P 3=\frac{\partial^{2}}{\partial y \partial z}\left[\overline{\left(v_{L}^{\prime}+v_{S}^{\prime}\right)\left(v_{L}^{\prime}+v_{S}^{\prime}\right)}-\overline{\left(w_{L}^{\prime}+w_{S}^{\prime}\right)\left(w_{L}^{\prime}+w_{S}^{\prime}\right)}\right] \\
P 4=\left(\frac{\partial^{2}}{\partial z^{2}}-\frac{\partial^{2}}{\partial y^{2}}\right)\left[\overline{\left(v_{L}^{\prime}+v_{S}^{\prime}\right)\left(w_{L}^{\prime}+w_{S}^{\prime}\right)}\right]
\end{gathered}
$$

The terms analyzed in detail in this work are $P 3$ and $P 4$, which can be decomposed into a large scale $\left(P 3_{L}, P 4_{L}\right)$, a small scale $\left(P 3_{S}, P 4_{S}\right)$ and an interscale $\left(P 3_{L S}, P 4_{L S}\right)$ contribution as

$$
\begin{gathered}
P 3_{L}=\frac{\partial^{2}}{\partial y \partial z}\left[\overline{\left(v_{L}^{\prime 2}\right)}-\overline{\left(w_{L}^{\prime 2}\right)}\right] \\
P 3_{S}=\frac{\partial^{2}}{\partial y \partial z}\left[\overline{\left(v_{S}^{\prime 2}\right)}-\overline{\left(w_{S}^{\prime 2}\right)}\right] \\
P 3_{L S}=2 \frac{\partial^{2}}{\partial y \partial z}\left[\overline{\left(v_{L}^{\prime} v_{S}^{\prime}\right)}-\overline{\left(w_{L}^{\prime} w_{S}^{\prime}\right)}\right] \\
P 4_{L}=\left(\frac{\partial^{2}}{\partial z^{2}}-\frac{\partial^{2}}{\partial y^{2}}\right)\left[\overline{v_{L}^{\prime} w_{L}^{\prime}}\right] \\
P 4_{S}=\left(\frac{\partial^{2}}{\partial z^{2}}-\frac{\partial^{2}}{\partial y^{2}}\right)\left[\overline{v_{S}^{\prime} w_{S}^{\prime}}\right] \\
P 4_{L S}=\left(\frac{\partial^{2}}{\partial z^{2}}-\frac{\partial^{2}}{\partial y^{2}}\right)\left[\overline{\left(v_{L}^{\prime} w_{S}^{\prime}+v_{S}^{\prime} w_{L}^{\prime}\right)}\right]
\end{gathered}
$$




\section{B. Definition of Corner Vortex}

To anticipate one of the observations presented subsequently, the instantaneous velocity field in the corner is characterized by multiple small-scale vortices embedded in a large-scale component that is part of the corner flow. The scale decomposition helps to differentiate between the two components. Thus, to avoid ambiguities, we here attempt at formally and mathematically defining the corner vortex pair. This definition will then be used to extract what are here defined the corner vortex pair from each instantaneous image and perform a conditional statistical analysis of its properties.

The instantaneous velocity vector fields (full and scale decomposed fields) obtained from SPIV planes were decomposed into rigid rotation vorticity, pure straining and pure shear using Triple Decomposition of Motion (TDM) [29, 30]. Rigid rotation vorticity $\left(\omega_{R R}\right)$ is an alternative vortex identification scheme and provide similar information of other methods, but the added benefit that the sign is preserved and can be linked directly to vorticity. Since we apply the definitions on the large scale component field $\left(\mathbf{u}_{L}\right)$, we here define the method based on this component and the corresponding $\omega_{R R_{L}}$ extracted from $\mathbf{u}_{L}$. ( $\omega$ is here used to indicate the streamwise component of a vorticity since in our measurements we only have access to the streamwise component.) The planar projection of an instantaneous vortex tube $(\varphi)$ referred to as vortex henceforth is defined as the area enclosed $(A)$ by an iso-contour closed loop $\left(\left|\omega_{R R_{L}}\right|=10\right)$. The centroid of the iso-contour closed loop defines the vortex center $(\tilde{y}, \tilde{z})$ for TV planes. This was then used to define a corner vortex as follows.

Suppose an instantaneous vector field $m$ is characterized by a set $\phi$ of $n$ distinct vortices, $\phi=\left[\varphi_{1}, \varphi_{2}, \ldots, \varphi_{k}, \ldots \varphi_{n}\right]$, an ordered set $\alpha$ of corresponding areas $\phi=\left[A_{1}, A_{2}, \ldots, A_{k}, \ldots A_{n}\right]$ and a set of corresponding ordered pairs of vortex center co-ordinates $V C=\left[\left(\tilde{y}_{1}, \tilde{z}_{1}\right),\left(\tilde{y}_{2}, \tilde{z}_{2}\right), \ldots,\left(\tilde{y}_{k}, \tilde{z}_{k}\right), \ldots\left(\tilde{y}_{n}, \tilde{z}_{n}\right)\right]$. The negative corner vortex $\left(\Phi_{-}\right)$of the instantaneous vector field $m$ is defined as follows:

$$
\begin{array}{r}
\Phi_{-}:=\left\{\varphi_{k} \mid \varphi_{k} \in \phi, \oint_{A_{k}} \omega_{R R_{L}} d A_{k}<0, \oint_{A_{k}} \omega_{R R_{L}} d A_{k}=\right. \\
\left.=\min \left[\oint_{A_{n}} \omega_{R R_{L}} d A_{n} \mid \varphi_{n} \in \phi\right], \tilde{y_{k}} \leq \delta, \tilde{z_{k}} \leq 1.5 \delta, \tilde{z_{k}}>K \tilde{y_{k}}\right\}
\end{array}
$$

where $\mathrm{K}$ is the aspect ratio of the wind tunnel defined by $\left(K=z_{T} / y_{T}\right)$ and $d A_{k}$ is the differential area element in the area enclosed by the the vortex tube boundary of $k^{t h}$ vortex. Similarly the positive corner vortex $\left(\Phi_{+}\right)$of vector field $m$ is defined as:

$$
\begin{array}{r}
\Phi_{+}:=\left\{\varphi_{k} \mid \varphi_{k} \in \phi, \oint_{A_{k}} \omega_{R R_{L}} d A_{k}>0, \oint_{A_{k}} \omega_{R R_{L}} d A_{k}=\right. \\
\left.=\max \left[\oint_{A_{n}} \omega_{R R_{L}} d A_{n} \mid \varphi_{n} \in \phi\right], \tilde{y_{k}} \leq 1.5 \delta, \tilde{z_{k}} \leq \delta, \tilde{z_{k}}<K \tilde{y_{k}}\right\}
\end{array}
$$

If no vortices satisfying the above definitions are found in an instantaneous field, that field is discarded from further statistical analyses. The characteristic length $\lambda$ of a corner vortex $\Phi$ is defined as

$$
\lambda:=\sqrt{A_{\Phi}}
$$

where $A_{\Phi}$ is the area of corner vortex $\Phi$.

An example of instantaneous rigid rotation vorticity field is shown in Figure 3. The black solid line $z=K y$ represents the diagonal of the wind tunnel rectangular channel. Thus the center belonging to the negative corner vortex must lie above this line as per the definition 26 while the center belonging to the positive corner vortex must lie below it (definition 27). The vertical dashed red line $(y=1.5 \delta)$ and horizontal red dashed line $(z=\delta)$ are the vertical and horizontal bounds below $z=K y$ that bound the region of existence of the positive corner vortex. Similarly the vertical blue dashed line $(y=\delta)$ and horizontal blue dashed line $(z=1.5 \delta)$ are the vertical and horizontal bounds above $z=K y$ for the region of existence of negative corner vortex. Thus the vortex marked $\Phi_{-}$forms the negative corner vortex with center $\left(\tilde{y}_{-}, \tilde{z}_{-}\right)$and the vortex marked $\Phi_{+}$forms the positive corner vortex with center $\left(\tilde{y}_{+}, \tilde{z}_{+}\right)$as these are the strongest and largest of the vortices lying in their respective domains. 
If $\mathbf{r}_{+}=\left(\tilde{y}_{+}, \tilde{z}_{+}\right)$and $\mathbf{r}_{-}=\left(\tilde{y}_{-}, \tilde{z}_{-}\right)$represent the position vectors of the positive and negative corner vortex centers for the given instantaneous vector field $m$, the corner vortex separation distance $(\gamma)$ is defined by

$$
\gamma=\left|\mathbf{r}_{+}-\mathbf{r}_{-}\right|
$$

The position vector of center of circulation $\mathbf{r}_{\mathbf{c e n}}$ for the corner vortex pair $\Phi_{+}$and $\Phi_{-}$is defined as

$$
\mathbf{r}_{c e n}=\frac{\mathbf{r}_{+}\left(\oint_{A_{+}}\left|\omega_{R R_{+L}}\right| d A_{+}\right)+\mathbf{r}_{-}\left(\oint_{A_{-}}\left|\omega_{R R_{-L}}\right| d A_{-}\right)}{\left(\oint_{A_{+}}\left|\omega_{R R_{+L}}\right| d A_{+}\right)+\left(\oint_{A_{-}}\left|\omega_{R R_{-L}}\right| d A_{-}\right)}
$$

The corner bisector angle $\theta_{b i s}$ is then defined as

$$
\theta_{b i s}=\arg \left(\mathbf{r}_{c e n}\right)
$$

\section{Analyses, Results and Discussions}

\section{A. General instantaneous flow characteristics}

An example of instantaneous velocity fields from each of the three dataset locations is shown in Figure 4 . The in plane velocity fields are shown as vectors $(v \hat{j}+w \hat{k})$ while the out of plane velocity component $(u)$ is shown by color contours. The $y=0$ line represents the side-wall and the $z=0$ line represents the bottom-wall. The flow near the corners is turbulent and characterized by eddies which will be clearer in the vorticity fields in subsequent sections. We can observe that at $x=-100 \mathrm{~mm}$ location, high momentum flow dominates most of the interrogation region as shown in Figure 4a. As we proceed downstream, we can see from Figure 4b and Figure 4c that a low momentum layer is formed near the walls. A strong secondary flow is observed in Figure $4 \mathrm{c}$ away from the side-wall at a height of about $10 \mathrm{~mm}$ from the bottom-wall which convects the low momentum side-wall boundary layer fluid towards the core flow.

\section{B. Mean primary flow field}

The mean flow fields obtained from uncorrelated sequence of images obtained at the same locations are shown in Figure 5. Figure 5a shows the streamwise velocity contours of the most upstream data-plane with the streamwise flow being out of the plane. It can be seen that the boundary layer thickness, defined by the $99 \%$ iso-contour line, is fairly uniform without any curvature indicating that the flow is fairly symmetric relative to the corner at this location. Also the close proximity of the sonic line to the side-wall indicates that the flow is mostly supersonic throughout the crosssection of the tunnel at $x=-100 \mathrm{~mm}$. The sonic line was computed using the local speed and assuming a calorifically perfect gas with unit Prandtl number and a $T_{\infty} / T_{w}$ value of 0.37 for Mach 2.75 by interpolating the experimental values compiled by Spina et al. [31].

It can be seen from Figure $5 \mathrm{~b}$ (plane at $x=-50 \mathrm{~mm}$ ) that the side-wall $99 \%$ boundary layer thickness has started to thicken towards the bottom-wall before it merges with the bottom-wall boundary layer. On the contrary, the boundary layer on the bottom-wall remains fairly undisturbed. The sonic line now extends along the entire walls of the tunnel. Also a fair portion of the flow is subsonic at the corner. It can be observed that the subsonic flow is biased towards the side-wall rather than the bottom-wall, probably due to the one-sided nature of the nozzle.

Further downstream at TV3 $(x=75 \mathrm{~mm})$, it is to be noted that boundary layer flow now occupies nearly the entire domain of interrogation. Low momentum region (yellow zone of the contour) can be seen bulging out (at a height of about $10 \mathrm{~mm}$ from the bottom-wall) from the side-wall towards the core flow indicating a momentum transport due to secondary flow. At the same time a high momentum region (red zone of the contour) can be seen penetrating towards the corner. This effect of curvature of iso-tach (constant velocity) lines has been observed by Gessner et al. [1, 5], Brundrett and Baines [10] and associated with underlying vortical momentum transport by Perkins [9]. It is to be noted that the sonic line in this case tends to conform to both the walls unlike TV2 possibly indicating spatial unsteadiness of the secondary flows responsible for subsonic momentum transport, which would be masked by averaging. 
Another important conclusion that can be drawn from Figure 5 is that the observed flow field is asymmetric unlike the symmetric/anti-symmetric flow fields about the corner bisector discussed in previous studies $[1,4,5,10,9]$. The primary reason for this irregularity is suspected to be the one sided nozzle and the low aspect ratio characteristic of the wind tunnel.

Figure 6 shows the velocity profiles of average streamwise velocity $U$ perpendicular to the bottom and side-walls. As is expected the flow at the most upstream location TV1 sports a near canonical mean velocity profile (see also [28]) without any visible inflection points and seems to be similar to that of an attached flow (see $y=5 \mathrm{~mm}$ and $z=5 \mathrm{~mm}$ in Figure 6a and Figure 6b). At the downstream location $x=-50 \mathrm{~mm}$, it can be seen from Figure $6 \mathrm{c}$ and Figure $6 \mathrm{~d}$ that the velocity profile now sports an inflection point near the corner $(y=5 \mathrm{~mm}$ and $z=5 \mathrm{~mm})$. Also the velocity profile at the location of TV2 near the corner $y=5 \mathrm{~mm}$ and $z=5 \mathrm{~mm}$ sports a velocity defect compared to the profiles away from the corner as seen in Figure $6 \mathrm{c}$ and Figure 6d. The mean flow profile at the downstream TV3 location shows similar characteristics to the flow at TV2 location with a relatively higher velocity defect in the profile at $y=5 \mathrm{~mm}$. Further downstream at $x=75 \mathrm{~mm}$ where the secondary flow (see section C) is relatively well developed, it starts affecting the mean primary flow which can be seen in the velocity profiles. The streamwise velocity profile at $y=5 \mathrm{~mm}$ (Figure $6 \mathrm{e}$ is seen to have a minimum point at about $z=8 \mathrm{~mm}$ indicating that momentum transport occurs).

\section{Mean secondary flow field}

Contour plots of the transverse (in-plane) velocity components are shown in Figure 7 and Figure 8. Although the streamwise velocity field at $x=-100 \mathrm{~mm}$ is fairly uniform, a study of the transverse velocity field reveals the presence of a weak secondary flow in proximity of the corner as is seen in Figure 7a and Figure 8a. The V-component at these locations is directed towards the corner in the immediate vicinity of the corner and then reverses direction away from corner along the side-wall and bottom-wall. As we proceed downstream the magnitude of this negative V-region initially increases at $x=-50 \mathrm{~mm}$ and then decreases at $x=75 \mathrm{~mm}$, however the size of the region increases as seen from Figure $7 \mathrm{c}$ respectively, indicating a possible increase in spatial unsteadiness of secondary flows at these locations.

\section{Large and small scale velocity fields}

As can be seen from the original fields the flow near the walls is turbulent and characterized by small scale eddies. The vector fields are decomposed into large scale and small scale fields by the use of a spatial averaging filter spanning ( $24 \times 24$ pixels) which is equivalent to a characteristic length of $3.65 \mathrm{~mm}$ or $\delta_{99} / 2$ at the location of TV1.

Instantaneous vector fields at the large scales obtained by decomposing the instantaneous flow field shown in Figure 4 are shown in Figure 9. The large scale swirling motion is more discernible from the in plane vector fields as compared to the original fields (Figure 4). We can observe that as we go downstream the swirling motion in the instantaneous field is stronger and larger. The background contour of the streamwise velocity component is smoother but similar to the original field. The large scale flow at the most downstream location (TV4) is observed to be dominated by a strong negative vortex which seems to be responsible for the momentum transport in the corner. A bulge of low momentum region is observed stretching away from the side-wall towards the core flow from the location where this vortex is drawing low momentum fluid from the near side-wall fluid flow. The instantaneous small scale velocity fields (Figure 10) associated with the described instant showed no discernible structure, but are characterized by a forest of small scale vortices. At this time, analyses of the small scale fields is omitted.

Mean flow fields at different scales were obtained by averaging multiple uncorrelated instances at the corresponding scales. The mean large scale velocity fields are shown in Figure 11. The mean large scale fields follow the same general trend as the mean complete fields in Figure 5, however they are characterized by a thicker subsonic region which is an artifact of spatial filtering near the wall used in decomposition. Mean small scale velocity fields are shown in Figure 12. Comparing the amplitudes in Figures 11 and 12, it can be observed that the small scale fields are at a much lower amplitude (less than 10\%) as compared to the large scale velocity fields. The streamwise momentum transport due to large scale secondary flows is however much more pronounced in the small scale velocity fields. Pockets of negative streamwise small scale velocity component can be observed in Figures 12a and 12b along the 
side-wall at about $z=5 \mathrm{~mm}$ and along the bottom-wall at about $y=10 \mathrm{~mm}$. These are the locations from where the secondary flows draw the lower momentum fluid from near the walls towards the core flow. Further downstream (Figure 12c) at the location of TV3 $(x=75 \mathrm{~mm})$ the regions of negative streamwise small scale velocities move away from the corner with the pocket of negative streamwise velocity along the side-wall growing in size and strength. A strand of positive streamwise velocity is observed in between the regions of streamwise negative velocity extending away from the corner. This region corresponds to the momentum transport due to the large scale secondary flows from the core towards the corner.

The mean large scale in plane velocity $\left(V_{L}, W_{L}\right)$ fields have a similar structure with slightly weaker magnitudes with respect to the complete fields (Figures 7 and 8 ). The mean small scale in plane velocity fields show no discernible structure and are characterized by velocity amplitudes to the order of about $0.01 U_{\infty}$, which is within the uncertainty of the measurements.

\section{E. Vortex fields}

Because the flow in the corner is dominated by heavy shear and strain, traditional Cauchy Stokes' decomposition of motion into symmetric part (shear) and anti-symmetric (vorticity) is insufficient. The shear and strain mask the vorticity associated with vortex tubes and makes identifying the corner vortices difficult. Thus, a planar surrogate of Triple Decomposition of Motion (TDM) developed by Kolar [29] was used to determine only the part of vorticity associated with rigid-body rotation $\left(\omega_{R R}\right)$, which makes identifying the vortex cores clearer.

\section{Intantaneous vortex fields}

Figure 13 shows the instantaneous rigid rotation vortex fields obtained from the instantaneous velocity fields shown in Figure 4. As we can see no large scale distinctive corner vortex system discussed in the previous studies [4, 5, 1, 10, 9] is observed in instantaneous fields. Instead, the flow within boundary layer near the corner is dominated by a forest of vortices or vortex tubes if a complete $3 \mathrm{D}$ picture is considered. The vortices tend to spread away from the walls as we proceed downstream due to boundary layer growth.

Applying TDM to the large scale velocity fields presents a clear picture of the large scale vortices present in the corner. The large scale instantaneous rigid rotation vorticity fields are shown in Figure 14. The rigid rotation vorticity field is now dominated by a few large scale vortices, the locations and direction of which resemble those described in the previous studies (Section II). In the downstream planes of TV2 and TV3 where the secondary flow is relatively well developed, a pair of anti-symmetric vortices are observed that convect the high momentum fluid from the core flow towards the corner. In TV2 (Figure 14b) a large scale negative vortex belonging to the corner system as defined in Section B is observed near the side-wall centered at about $z=6 \mathrm{~mm}$ along with a positive corner vortex centered at about $y=12 \mathrm{~mm}$ along the bottom-wall. A similar layout can be observed in the TV3 plane, however the instance presented shows a weaker positive corner vortex unlike the instance showed in Figure 3 showing a typical corner rigid rotation vorticity field at TV3. Small scale rigid vorticity fields are comprised of a forest of smaller vortex cores similar to the rigid rotation vorticity fields shown in Figure 13.

\section{Mean vortex fields}

Rigid rotation vorticity fields obtained from the complete mean flow fields of Figure 5 are given in Figure 15. It can be observed that an anti-symmetric (with respect to sign) vortex system can be found conforming to the side-wall (negative vortex) and the bottom-wall (positive vortex). Although this system has signs consistent with a canonical corner vortex system, its location is fairly displaced from the corner. Moreover this system is geometrically asymmetric, which may be due to the asymmetric nature of the wind tunnel. At the most upstream location of TV1, we can observe from Figure 15a that a negative vortex exists along the side-wall at about $z=4 \mathrm{~mm}$ while traces of positive vortex exist along the bottom-wall around $y=6 \mathrm{~mm}$. As we proceed downstream to $x=-50 \mathrm{~mm}$, it can be seen from Figure $15 \mathrm{~b}$ there is a slight increase in the strengths of the negative and positive vortices which remain fairly at the 
same location. At the location of $x=75 \mathrm{~mm}$ the traces of negative vortex can be seen at about $z=5 \mathrm{~mm}$ (15c) while there exist only a few weak traces of the positive corner vortex. The general lack of strong corner vortices identified by the averaging process is attributed to the unsteady nature of the vortex system that washes out the mean structure of the vortex pair.

An alternative way to evaluate the average location of vortices is to compute the mean value of the instantaneous rigid rotation vorticity fields (i.e., by averaging fields like that of Figure 14 rather than computing $\omega_{R R}$ from the mean fields of Figure 11 to obtain the fields of Figure 15). Fields obtained in this way are shown in Figure 16, but do not match the vorticity fields computed from the mean flow (the TDM does not commute). We can observe from Figure 16a that an anti-symmetric (both in sign as well as geometry) vortex system is likely to exist in the corner, but its structure thus identified is not consistent with the canonical corner vortex pair. In fact the distribution of $\omega_{R R}$ is not concentrated in a well defined region, but it spreads over the entire corner region, although symmetry in the sign (negative above and positive below the corner bisector) is maintained. We attribute this property to an inherent unsteadiness of the corner vortex system. Marching downstream, it can be seen from Figure 16b that the anti-symmetric vortex system has increased in size since the corner region occupied by $\omega_{R R}$ has increased.

Mean vorticity field obtained by averaging rigid rotation fields of large scale velocity fields are shown in Figure 17. The scale decomposition allow us to more clearly identify the existence of the corner vortex system. Distinct negative and positive zones of vorticity associated with the corner system can be observed. In TV1 and TV2 (Figures 17a $\& 17 \mathrm{~b})$ there exists a region of mean negative vorticity near the corner along the side-wall at about $z=3 \mathrm{~mm}$ and the positive vorticity associated with the corner system is found along the bottom-wall in the range $y=5-10 \mathrm{~mm}$. Further downstream at TV3 (Figure 17c), it can be observed that the negative vorticity region associated with the corner vortex system has grown in size which may indicate constrained spatial unsteadiness while the region associated with the positive corner vortex has significantly decreased in size which may be due to increased unsteadiness.

\section{Conditional sampling and corner vortex statistics}

Centers of the corner vortices were determined as per described in section B for each instantaneous vector field after the scale decomposition was applied. Conditional averaging of instantaneous (large scale) vector fields was conducted in a two step process. First, an average field was constructed in a reference frame shifted to the center of the negative or positive corner vortex; thus generating two mean fields, one as seen from the center of the negative corner vortex and the other from the center of the positive corner vortex. Then, the resulting field was shifted back to the original frame of reference by the average position of each of the two vortexes in the pair. These conditionally averaged vector fields are shown in Figures 18, 19 and 20 for the three planes. The conditionally averaged fields provide a relative measure of how the flow looks like around the corner vortex, its scaling and the average strength.

Figure 18a shows the average vector field in the vicinity of the negative corner vortex at $x=-100 \mathrm{~mm}$. The rigid rotation vorticity and conventional vorticity fields associated with the vector field in Figure 18a are shown in Figures $18 \mathrm{~b}$ and $18 \mathrm{c}$. The circular motion created by the negative corner vortex is well observed in the vector field. The mean position of the vortex center at this location as observed from the instantaneous vector field statistics is $\left(\bar{y}_{n}, \bar{z}_{n}\right)=(2.63,4.64) \mathrm{mm}$. Similar properties of the positive and negative corner vortex can be measured at different data plane locations and are tabulated in Table 2. Note also that the process of conditionally averaging for the detection of the positive or negative vortex reinforces only the intended vortex, the resulting conditionally averaged field still maintain a signature, albeit weak, of the opposite vortex. As we proceed downstream we can see (Figure 19 and 20) that both the corner vortices move away from the corner while their scales remain more or less constant with a slight increasing trend in the streamwise direction which is also evident from Table 2.

The probability density functions (PDF) of the location of corner vortex centers are shown in Figure 21. The most probable location of the positive corner vortex lies between the bottom-wall and $z=5 \mathrm{~mm}$. It moves away from the corner along the bottom-wall in the downstream direction. The negative corner vortex has a high probability of residing in the range of $z=0-5 \mathrm{~mm}$ along the side-wall at the first two locations (TV1 and TV2), while at TV3 it appears to become spatially unstable with a larger standard deviation. The PDFs of the corner bisector angle $\left(\theta_{b i s}\right)$ and the corner vortex separation distance $(\gamma)$ are shown in Figure 22. It is worth noting that both the PDFs remain more or 
less the same at all three streamwise locations. The mean angle of corner bisector stays at about $40^{\circ}$, which is a value approximately equal to $\tan ^{-1}\left(y_{T} / z_{T}\right)$, which is the angle of the diagonal of the rectangular cross-section of the test section. The mean distance between the corner vortex pair stays constant roughly at $6.7 \mathrm{~mm}$.

\section{F. Strain rates and vortex-induced momentum transport}

In-plane strain rate components associated with the streamwise velocity are shown in Figure 23. The in-plane streamwise velocity gradients associated with the large scale fields follow a qualitatively similar but smoother pattern of what shown in Figure 23. The in-plane streamwise velocity gradient contour plots are superimposed by iso-contour lines of $\omega_{R R_{L}}$, with black lines representing the positive vorticity while white lines representing the negative vorticity. It can be observed that in all three locations the regions of high positive $\partial U / \partial z$ are confined near the bottom-wall and the thickness of these regions decreases towards the corner. A region of negative $\partial U / \partial z$ can be observed near the side-wall $(z=2-5 \mathrm{~mm}$ in TV1 and TV2 and around $z=4-10 \mathrm{~mm}$ in TV3) in the same region where the thickness of the region of positive $\partial U / \partial z$ along the bottom-wall starts to drop. It can be observed that mean large scale negative corner vortex (white iso-contour lines) is seen to reside in this negative region. The negative corner vortex convects lower momentum fluid away from the side-wall creating a decrease in the $U$-velocity as we traverse the positive $z$-direction from the bottom-wall near the side-wall. This effect is observed as a dip in the $U(z)$ profiles of Figure $6 e$ near the side-wall $(y=2.5 \& 5 \mathrm{~mm})$ and this produces a negative $\partial U / \partial z$ observed at the location of the negative vortex in Figure 23h. A similar effect is observed with the positive corner vortex near the bottom-wall. Since the $\partial U / \partial y$ field is positive at the location where $\partial U / \partial z$ is negative and vice versa, the product of $\partial U / \partial y$ and $\partial U / \partial z$ would identify the regions where momentum deficits occurs, refer to Figures $23 \mathrm{c}, 23 \mathrm{f} \& 23 \mathrm{i}$. To further explain this observation, consider as an example the case of TV3 where the secondary flows are most developed, and which presents the case of strongest vortex-induced momentum transport among the three planes. With reference to any vertical line near the side-wall $(y=0-5 \mathrm{~mm})$ in Figure 23i, the $U$-velocity profile (Figure 6e) increases with $z$ until the region with negative (blue) $(\partial U / \partial y)(\partial U / \partial z)$ is encountered, then it decreases until the end of the region of negative $(\partial U / \partial y)(\partial U / \partial z)$. The deficit in the streamwise momentum is highest at the end of the blue region and at the start of the positive (green/red) $(\partial U / \partial y)(\partial U / \partial z)$ region which is located at about $z=8 \mathrm{~mm}$ along the line $y=2.5 \mathrm{~mm}$. It can thus be observed from Figure 6e that the streamwise velocity profile reaches a minimum at about $z=8 \mathrm{~mm}$ and this identifies the location where momentum transport away from the wall due to the vortex is the strongest.

\section{G. Vorticity Production Fields}

As described in section A, the vorticity production terms are decomposed into large, small and interscales. Most of the vorticity production takes place in the large scales and interscale while the small scale production although of comparable magnitude lacks structure and appears random. Thus only the large and inter-scale vorticity production terms will be presented.

The vorticity production fields associated with large scale anisotropy are shown in Figure 24. It is observed that at TV1 $(x=-100 \mathrm{~mm})$ the field appears unstructured and weak as compared to the downstream planes where vorticity production takes place around the regions of high turbulence fluctuations. Vorticity production due to interscale anisotropy is stronger than that in the large scales and follows similar trend as can be observed in Figure 25.

The vorticity production caused by the cross diagonal components of large scale turbulence are shown in Figure 26. It can be observed that these fields are of the same orders of magnitude as the $P 3_{L}$ fields and are also primarily driven by the sites of higher values of turbulent fluctuations. It is worth noting that the anisotropic vorticity production fields and the fields of vorticity production due to cross diagonal turbulence terms are orthogonal to each other which was also observed by Brundrett and Baines[10]. Similar to the $P 3$ fields the contribution from the interscale terms is an order of magnitude higher than the large scale terms. 


\section{Conclusions}

A Mach 2.75 supersonic turbulent corner flow was experimentally investigated using stereo PIV. Three SPIV data planes orthogonal to the channel walls and the principal flow direction separated by a few boundary layer thicknesses were recorded. The data obtained was used for computing various parameters in order to provide an insight to the turbulent structures which would be characteristic of such a flow field. It was found that,

- No form of symmetry was evident from observing either instantaneous, mean velocity fields or vorticity fields obtained from mean velocity fields in the original form. Decomposing the vector fields into large and small scales revealed a skewed symmetry, skewed possibly due to the nature of the nozzle or the aspect ratio of the tunnel.

- The instantaneous flow fields consisted of a forest of vortices. Decomposing the velocity field into large and small scales made it possible to define the corner vortices and study their behavior in the streamwise direction. The average form of the corner vortices was made clear using conditional sampling.

- A statistical analysis revealed that the corner vortices move away from the corner while maintaining a similar probability density function of the inter-vortical distance and the corner bisector angle. The mean corner bisector angle at all the locations was found to be approximately equal to the characteristic angle of the channel aspect ratio. The mean characteristic scaling of the corner vortices was found to be of the same order of magnitude at all three locations with a slight tendency to increase downstream.

- The instantaneous vortex filaments possibly become increasingly unstable downstream as the Reynolds number increases. This is evident from decreasing amplitudes and increasing scales of mean vorticity structures observed in mean vorticity fields as well as the increasing standard deviations in the probable corner vortex center locations in vortex center location PDF fields.

- The effect of vortical momentum transport was observed in the iso-tach lines of streamwise velocity and the in plane strain rates associated with the streamwise velocity component.

- Gradients of large and inter-scale turbulence moments were found to be the major contributors to vorticity production with inter-scale turbulent moments contributing the most. The vorticity production fields due to turbulence anisotropy and the cross diagonal turbulence components were found to be orthogonal to each other which corroborates results of Brundrett and Baines[10].

\section{Acknowledgments}

The authors would like to thank Robin L. Klomparens, Dr. James Driscoll and Dr. Jack Benek for their valuable inputs. We also acknowledge Dr. Derek Dale for putting forth this $\mathrm{LT}_{\mathrm{E}} \mathrm{X}$ template.

\section{References}

[1] Gessner, F. B., "The origin of secondary flow in turbulent flow along a corner," Journal of Fluid Mechanics, Vol. 58, No. 1, 1972, pp. 1-25.

[2] Souverein, L. J., Dupont, P., Debiève, J.-F., Van Oudheusden, B. W., and Scarano, F., "Effect of Interaction Strength on Unsteadiness in Shock-Wave-Induced Separations," AIAA Journal, Vol. 48, No. 7, jul 2010, pp. 1480-1493.

[3] Morajkar, R. R., Klomparens, R. L., Eagle, W. E., Driscoll, J. F., Gamba, M., and Benek, J. A., "Relationship Between Intermittent Separation and Vortex Structure in a Low - Aspect Ratio 3D Shock Wave - Boundary Layer Interaction," Accepted for publication in AIAA Journal, pp. 1-52.

[4] Davis, D. O. and Gessner, F. B., "Further Experiments on Supersonic Turbulent Flow Development in a Square Duct," AIAA JOURNAL, Vol. 27, No. 8, 1989, pp. 1023-1030.

[5] Gessner, F. B., Eppich, H. M., and Lund, E. G., "Reynolds Number effects on near wall structure of turbulent flow along a streamwise corner," Near-Wall Turbulent Flows, 1993, pp. 965-975.

14 of 31

American Institute of Aeronautics and Astronautics 
[6] Ridha, A., "Flow along streamwise corners revisited," Journal of Fluid Mechanics, Vol. 476, mar 2003, pp. $223-265$.

[7] Rubin, S. G., “Incompressible flow along a corner,” Journal of Fluid Mechanics, Vol. 26, No. 01, mar 1966, pp. 97.

[8] Bradshaw, P., “Turbulent Secondary Flows," Annual Review of Fluid Mechanics, Vol. 19, No. 1, jan 1987, pp. 53-74.

[9] Perkins, H. J., "The formation of streamwise vorticity in turbulent flow," Journal of Fluid Mechanics, Vol. 44, No. 4, 1970, pp. 721-740.

[10] Brundrett, E. and Baines, W. D., "The production and diffusion of vorticity in duct flow," Journal of Fluid Mechanics, Vol. 19, No. 3, 1963, pp. 375- 394.

[11] Kornilov, V. I. and Kharitonov, a. M., "Investigation of the structure of turbulent flows in streamwise asymmetric corner configurations," Experiments in Fluids, Vol. 2, No. 4, 1984, pp. 205-212.

[12] Alizard, F., Robinet, J.-C., and Rist, U., "Sensitivity analysis of a streamwise corner flow," Physics of Fluids, Vol. 22, No. 1, 2010, pp. 014103.

[13] Zamir, M., "Similarity and stability of the laminar boundary layer in a streamwise corner," Proceedings of the Royal Society: Mathematical, Physical and Engineering Sciences, Vol. 377, 1981, pp. 269-288.

[14] Balachandar, S. and Malik, M. R., "Inviscid instability of streamwise corner flow," Journal of Fluid Mechanics, Vol. 282, apr 2006, pp. 187-201.

[15] Chapman, D. R., Kuehn, D. M., and Larson, H. K., "Investigation of separated flows in supersonic and subsonic streams with emphasis on the effect of transition," NACA Technical Report 1356, NACA, 1957, pp. 421-460.

[16] Kornilov, V. I., "Correlation of the separation region length in shock wave / channel boundary layer interaction," Experiments in Fluids, Vol. 23, No. January, 1997, pp. 489-497.

[17] Park, D. H., Park, S. O., Kwon, K. J., and Shim, H. J., "Particle Image Velocimetry Measurement of Laminar Boundary Layer in a Streamwise Corner,” AIAA Journal, Vol. 50, No. 4, apr 2012, pp. 811-817.

[18] Uruba, V., Hladík, O., and Jonáš, P., "Dynamics of Secondary flow in rectangular channel," Colloquium FLUID DYNAMICS 2011, Institute of Thermomechanics AS CR, Prague, 2011, pp. 1-7.

[19] Lapsa, A. P. and Dahm, W. J. A., "Stereo particle image velocimetry of nonequilibrium turbulence relaxation in a supersonic boundary layer," Experiments in Fluids, Vol. 50, No. 1, jun 2010, pp. 89-108.

[20] Eagle, W. E., Driscoll, J. F., and Benek, J. A., "3-D Inlet Shock-Boundary Layer Interactions - PIV Database for the Second SBLI Workshop," 30th AIAA Applied Aerodynamics Conference, No. June, American Institute of Aeronautics and Astronautics, New Orleans, LA, 2012.

[21] Eagle, W. E., Driscoll, J. F., and Benek, J. A., "Experimental Investigation of Corner Flows in Rectangular Supersonic Inlets with 3D Shock-Boundary Layer Effects," 49th AIAA Aerospace Sciences Meeting including the New Horizons Forum and Aerospace Exposition, No. January, American Institute of Aeronautics and Astronautics, Orlando, Florida, 2011, pp. 1-11.

[22] Eagle, W. E. and Driscoll, J. F., "Shock waveboundary layer interactions in rectangular inlets: three-dimensional separation topology and critical points," Journal of Fluid Mechanics, Vol. 756, sep 2014, pp. 328-353.

[23] Adrian, R. J., Christensen, K. T., and Liu, Z., "Analysis and interpretation of instantaneous turbulent velocity fields," Experiments in Fluids, Vol. 29, No. 95, 2000, pp. 275-290.

[24] Nogueira, J., Lecuona, A., and Rodriguez, P. A., "Data validation, false vectors correction and derived magnitudes calculation on PIV data," Measurement Science and Technology, Vol. 8, 1997, pp. 1493-1501.

[25] D’Errico, J., “inpaint_nans (Interpolation function)," Matlab Central File Exchange, Vol. https://www.mathworks.com/matlabcentral/fileexchange/4551-inpaint-nans.

[26] Moisy, F., "PIVMAT toolbox,” Vol. http://www.fast.u-psud.fr/pivmat/.

[27] Morajkar, R., Klomparens, R., Eagle, E., Driscoll, J., and Gamba, M., "Flow Separation Associated with 3 - D Shock Boundary Layer Interaction (SBLI)," SciTech 2014, American Institute of Aeronautics and Astronautics, Harbor Town, 2014, pp. 1-13.

[28] Morajkar, R. R., Driscoll, J. F., and Gamba, M., "Experimental study of supersonic turbulent corner flow evolution in a low aspect ratio rectangular channel,” AIAA SciTech2015, No. January, Kissimmee, Fl, 2015.

[29] Kolá, V., "Vortex identification: New requirements and limitations," International Journal of Heat and Fluid Flow, Vol. 28, No. 4, aug 2007, pp. 638-652. 
[30] Kolá, V., Šístek, J., Cirak, F., and Moses, P., “Average Corotation of Line Segments Near a Point and Vortex Identification," AIAA Journal, Vol. 51, No. 11, nov 2013, pp. 2678-2694.

[31] Spina E., Smits A., R. S., "The physics of supersonic turbulent boundary layers," Annual Review of Fluid Mechanics, Vol. 26, 1994, pp. 287-319.

16 of 31 


\section{Tables}

\begin{tabular}{llll}
\hline Mach number $M_{\infty}^{1}$ & $2.71 \pm 0.1$ & Static Temperature $T_{\infty}^{3}$ & $119 \pm 1 \mathrm{~K}$ \\
Mach number $M_{\infty}^{2}$ & $2.72 \pm 0.03$ & Test section height $^{4}$ & $69.3 \pm 0.2 \mathrm{~mm}$ \\
Free Stream velocity $U_{\infty}^{3}$ & $593 \pm 21 \mathrm{~m} / \mathrm{s}$ & Test section width $^{4}$ & $57.2 \pm 0.1 \mathrm{~mm}$ \\
Stagnation Pressure $P_{0}^{4}$ & $98.1 \pm 1 \mathrm{kPa}$ & Throat height $^{4}$ & $18.4 \pm 0.1 \mathrm{~mm}$ \\
Stagnation Temperature $T_{0}^{4}$ & $294 \pm 2 \mathrm{~K}$ & Throat to origin distance $^{4}$ & $481.5 \pm 1 \mathrm{~mm}$ \\
& \\
Wall Static Pressure $P(x=-85 \mathrm{~mm})^{4}$ & $4.15 \pm 0.6 \mathrm{kPa}$ & \\
\hline${ }^{1}$ Computed from a direct measure of stagnation and static pressure assuming isentropic expansion $(\kappa=1.4)$. \\
${ }^{2}$ Computed from a direct measure of the ratio of stagnation pressure and test section pitot pressure $(\kappa=1.4)$. \\
${ }^{3}$ Uncertainty estimated from error propagation rule. \\
${ }^{4}$ Uncertainty includes both measurement accuracy and day-to-day variability. \\
\hline
\end{tabular}

Table 1. Experimental conditions and their variabilities.

\begin{tabular}{ccccc}
\hline $\mathbf{x}(\mathbf{m m})$ location & $\left(\bar{y}_{p}, \bar{z}_{p}\right) \mathbf{m m}$ & $\left(\bar{y}_{n}, \bar{z}_{n}\right) \mathbf{m m}$ & $\bar{\lambda}_{p} \mathbf{m m}$ & $\bar{\lambda}_{n} \mathbf{m m}$ \\
\hline -100 (TV1) & $(8.42,3.76)$ & $(2.63,4.64)$ & 3.09 & 2.58 \\
-50 (TV2) & $(8.93,4.01)$ & $(2.57,4.92)$ & 3.1 & 2.74 \\
75 (TV4) & $(9.61,5.16)$ & $(4.26,5.67)$ & 3.86 & 3.05 \\
\hline
\end{tabular}

Table 2. Corner vortex properties at various streamwise locations. 


\section{Figures}

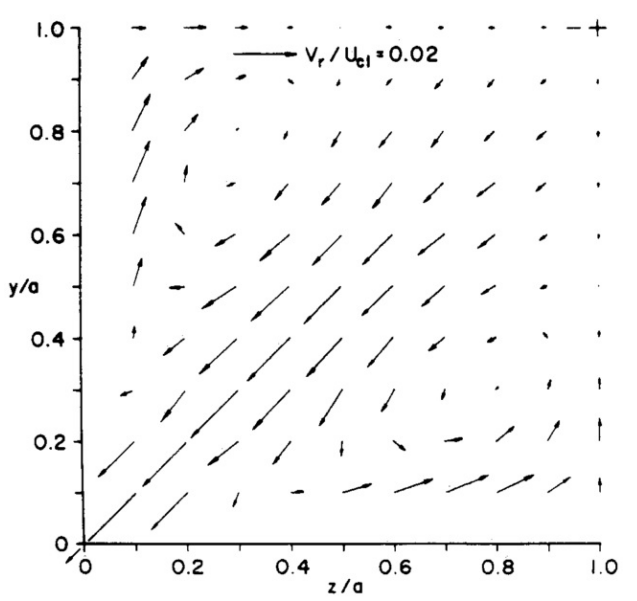

Figure 1. Proposed canonical flow structure(from Davis and Gessner [4]).

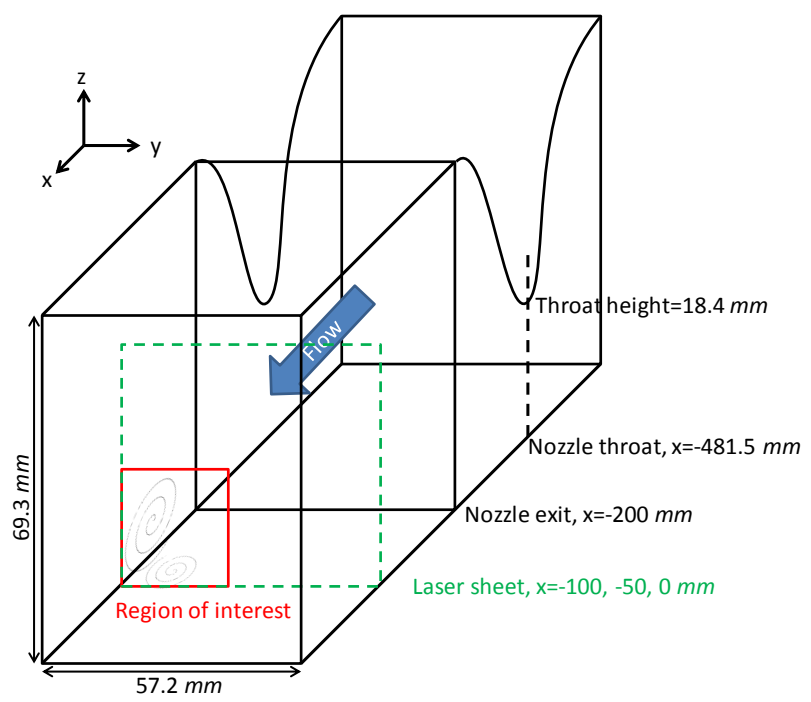

Figure 2. Wind tunnel schematic diagram (not to scale).

18 of 31 


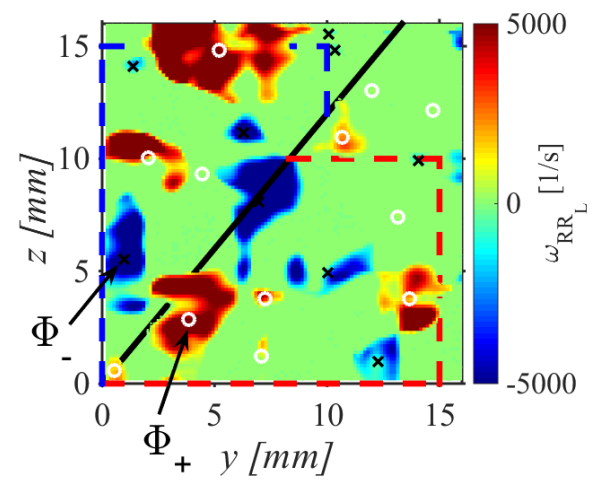

Figure 3. An instantaneous image in TV3 showing the vortex center detection and the pair of corner vortices as per definition. The region bounded by blue dashed lines and the solid black line is the domain of existence for the negative corner vortex. Similarly the region bounded by red dashed lines and the solid black line is the domain of existence for the positive corner vortex.

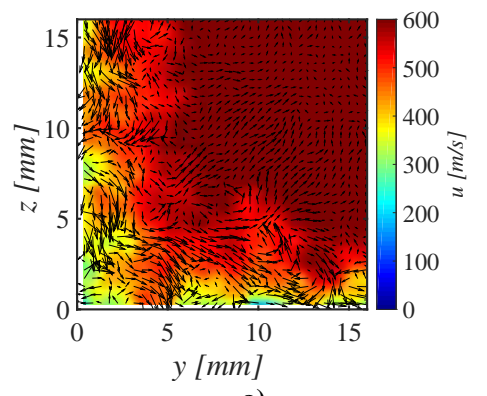

a)

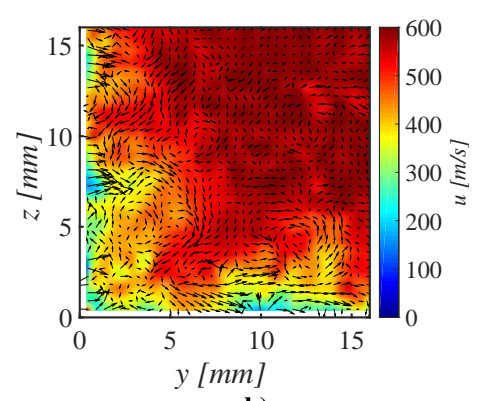

b)

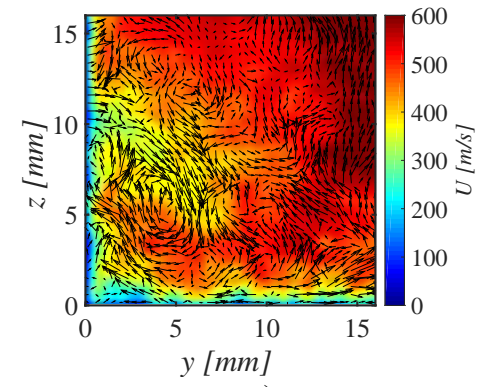

c)

Figure 4. Single instance of velocity field from 4a: TV1 $(x=-100 \mathrm{~mm}), 4 \mathrm{~b}:$ TV2 $(x=-50 \mathrm{~mm})$ and $4 \mathrm{c}$ : TV3 $(x=75 \mathrm{~mm})$. In plane velocity components are represented by vectors while the out of plane component is shown by the contour map.

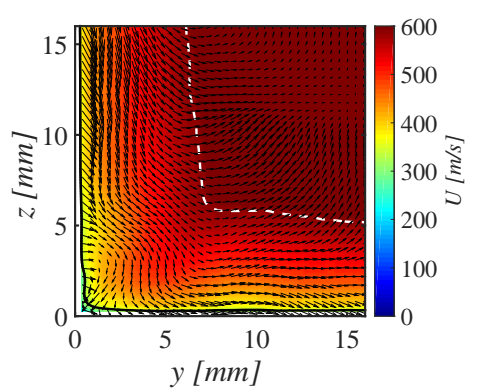

a)

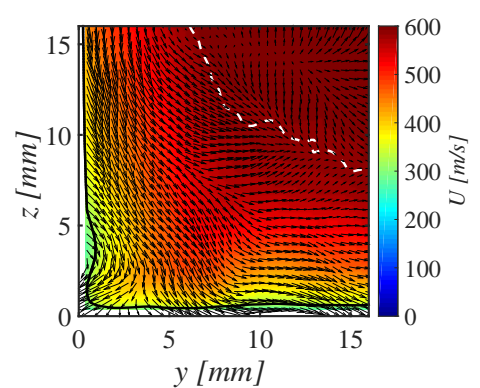

b)

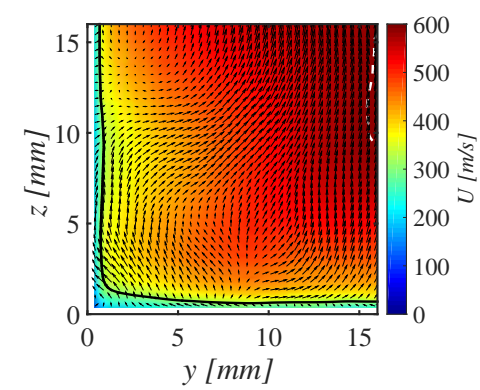

c)

Figure 5. Mean velocity field on 5a: TV1 $(x=-100 \mathrm{~mm})$; 5b: TV2 $(x=-50 \mathrm{~mm})$; and 5c: TV3 $(x=75 \mathrm{~mm})$. In-plane velocity components are represented by vectors while the out-of-plane component is shown by the contour map. The white dashed line represents the $\delta_{99}$ and the solid black line represents the contour of sonic velocity. 


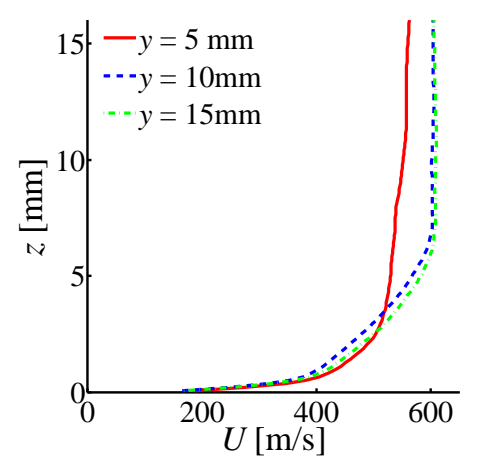

a)

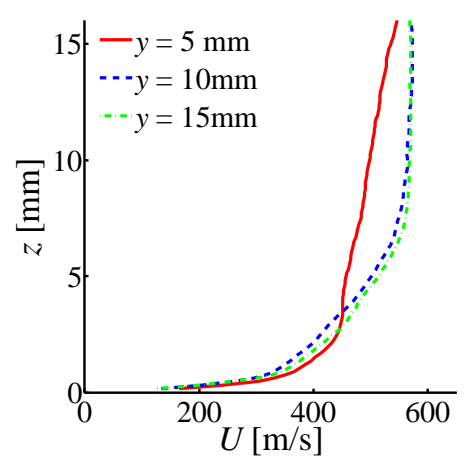

c)

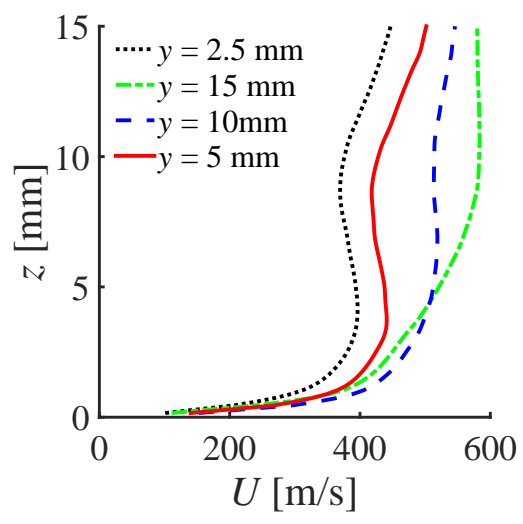

e)

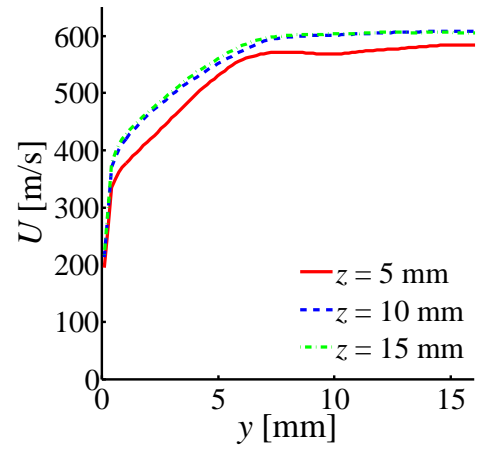

b)

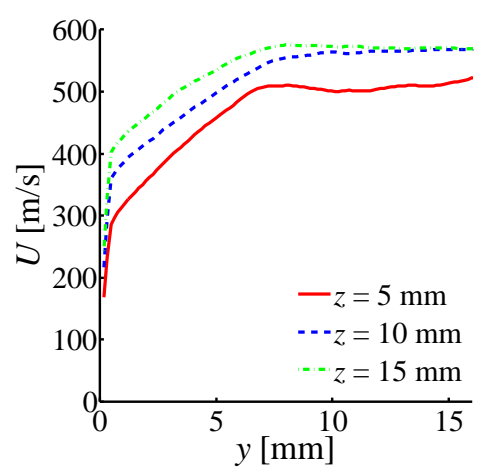

d)

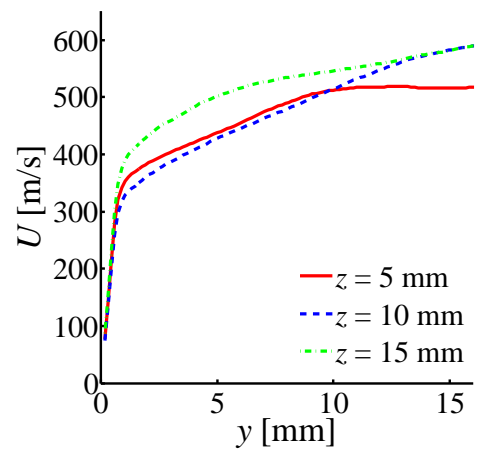

f)

Figure 6. Mean streamwise velocity component profiles from 6a: $U(z)$ on TV1; 6b: $U(y)$ on TV1; 6c: $U(z)$ on TV2; 6d: $U(y)$ on TV2; 6e: $U(z)$ on TV3; 6f: $U(y)$ on TV3. 


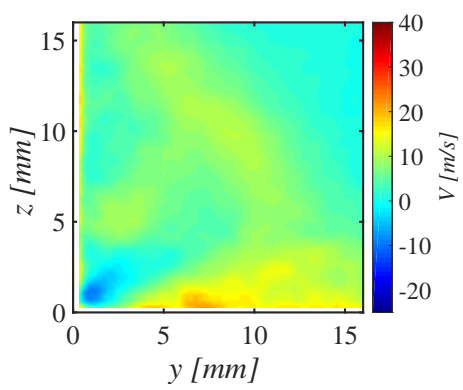

a)

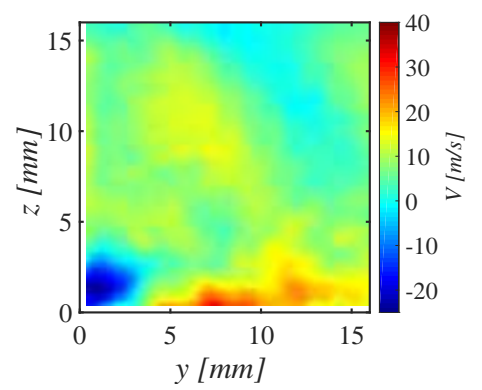

b)

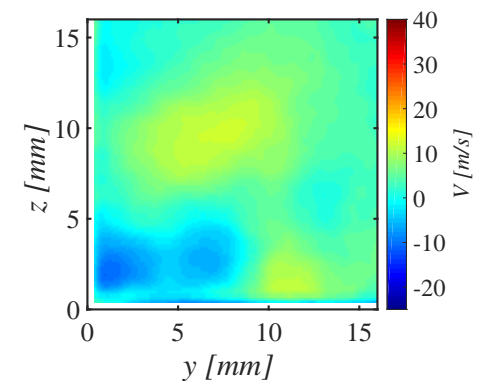

c)

Figure 7. Mean $V$ velocity components on different TV planes: $7 \mathrm{a}:$ TV1 $(x=-100 \mathrm{~mm})$, $7 \mathrm{~b}:$ TV2 $(x=-50 \mathrm{~mm})$ and $7 \mathrm{c}:$ TV3 $(\boldsymbol{x}=75 \mathrm{~mm})$.

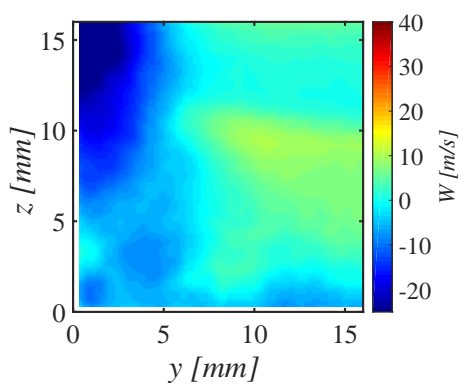

a)

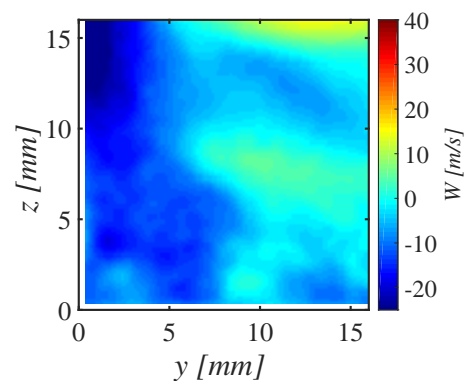

b)

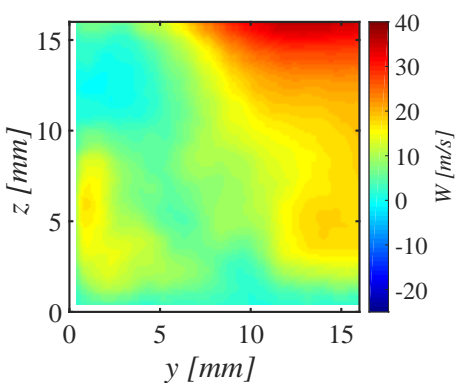

c)

Figure 8. Mean $W$ velocity components on different TV planes: $8 \mathrm{a}:$ TV1 $(x=-100 \mathrm{~mm}), 8 \mathrm{~b}:$ TV2 $(x=-50 \mathrm{~mm})$ and $8 \mathrm{c}$ : TV3 $(x=75 \mathrm{~mm})$.

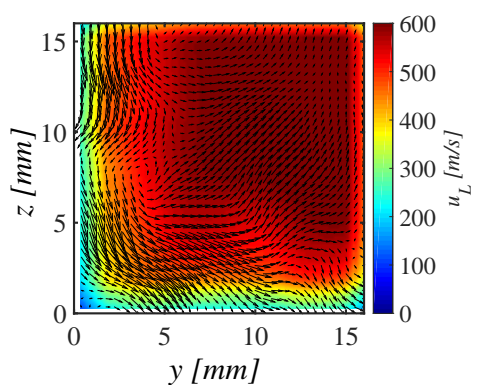

a)

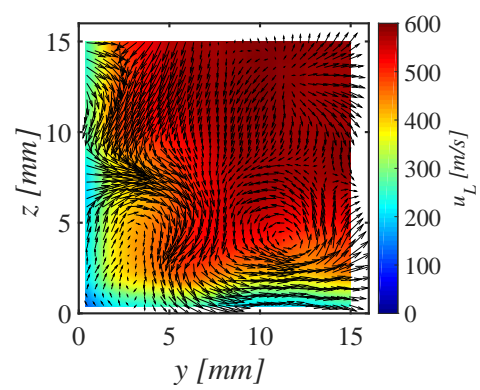

b)

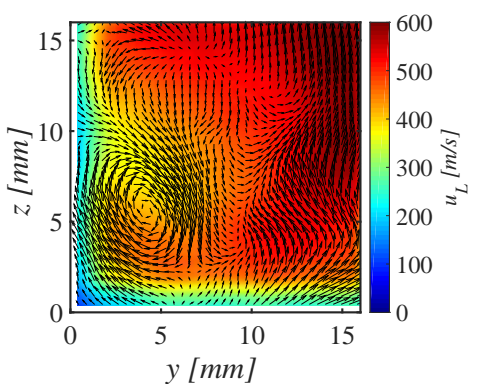

c)

Figure 9. Instantaneous large scale velocity vector fields obtained by decomposing instantaneous fields in Figure 4 at 9 a: TV1 $(x=$ $-100 \mathrm{~mm})$, 9b: TV2 $(x=-50 \mathrm{~mm})$ and 9c: TV3 $(x=75 \mathrm{~mm})$. 


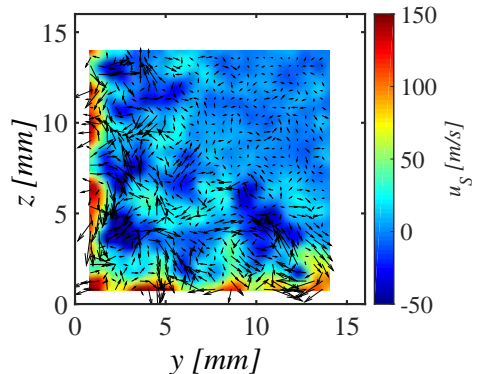

a)

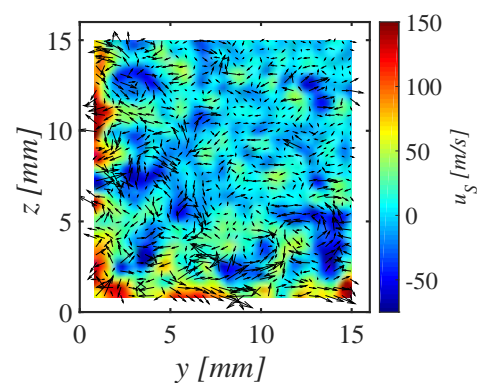

b)

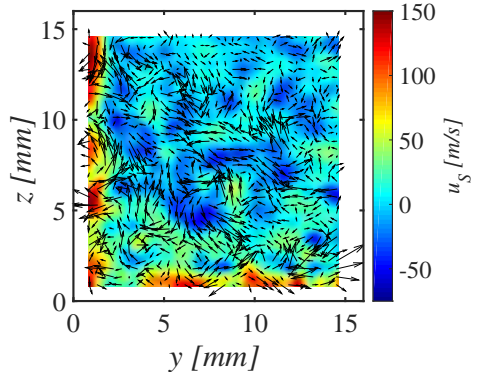

c)

Figure 10. Instantaneous small scale velocity vector fields obtained by decomposing instantaneous fields in Figure 4 at 10 a: TV1 $(x=$ $-100 \mathrm{~mm}), 10 \mathrm{~b}:$ TV2 $(x=-50 \mathrm{~mm})$ and 10c: TV3 $(x=75 \mathrm{~mm})$.

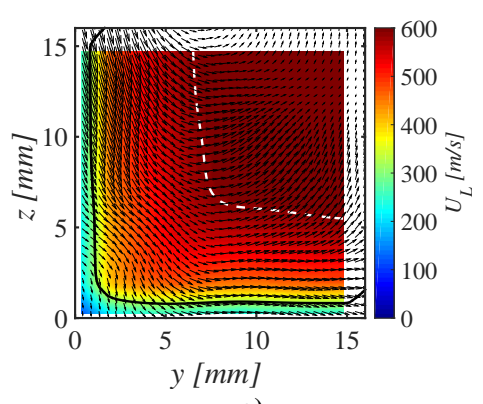

a)

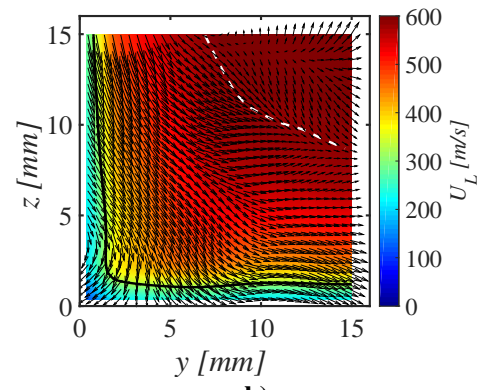

b)

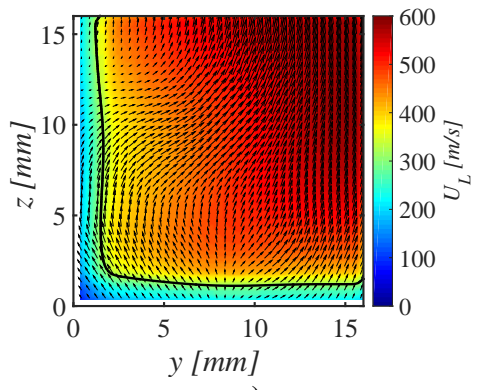

c)

Figure 11. Mean large scale velocity vector fields from 11a: TV1 $(x=-100 \mathrm{~mm}), 11 \mathrm{~b}:$ TV2 $(x=-50 \mathrm{~mm})$ and $11 \mathrm{c:} \mathrm{TV3}(x=75 \mathrm{~mm})$.

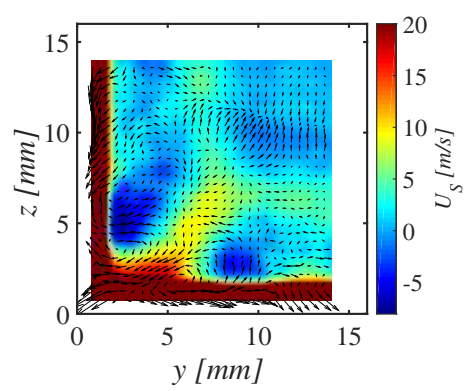

a)

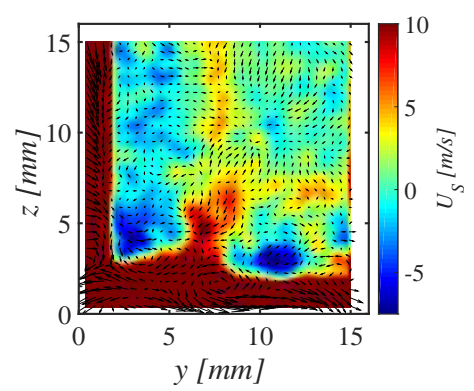

b)

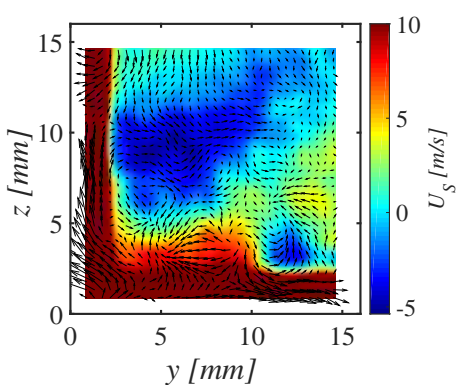

c)

Figure 12. Mean small scale velocity vector fields from 12a: TV1 $(x=-100 \mathrm{~mm}), 12 \mathrm{~b}:$ TV2 $(x=-50 \mathrm{~mm})$ and $12 \mathrm{c}:$ TV3 $(x=75 \mathrm{~mm})$. 


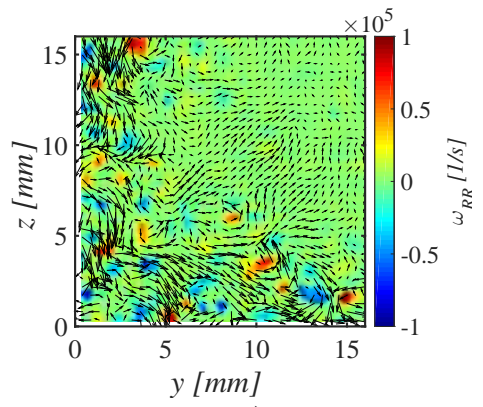

a)

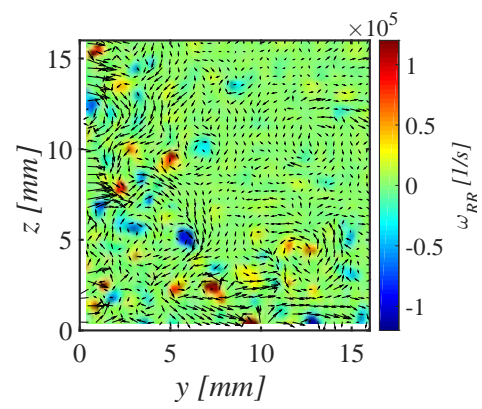

b)

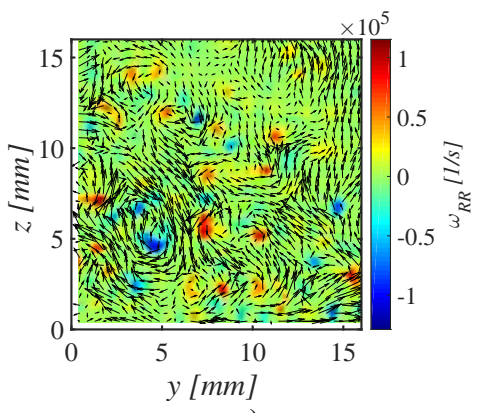

c)

Figure 13. Instantaneous rigid rotation vorticity field of instantaneous vector fields shown in Figure 4 from $13 a$ : TV1 $(x=-100 \mathrm{~mm})$, 13b: TV2 $(x=-50 \mathrm{~mm}) \& 13 \mathrm{c}: \operatorname{TV3}(x=75 \mathrm{~mm})$.

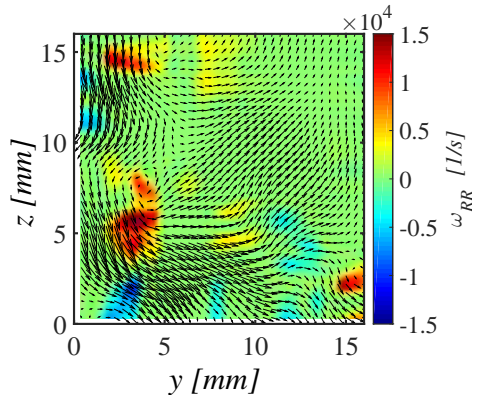

a)

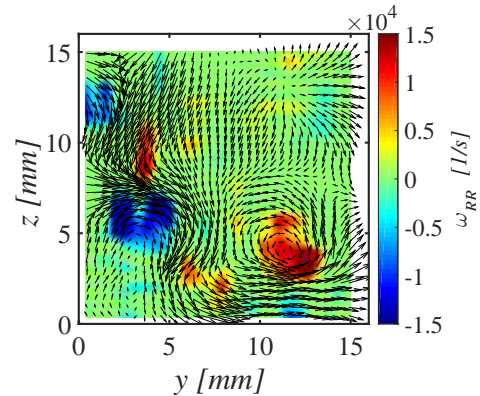

b)

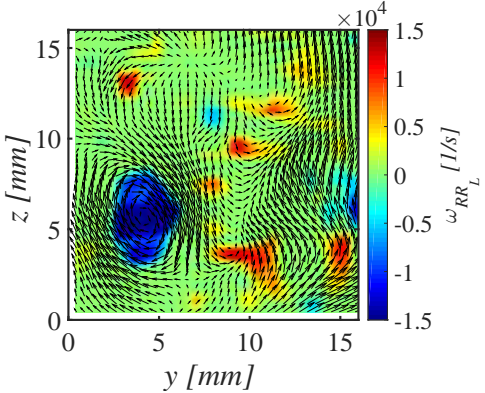

c)

Figure 14. Instantaneous rigid rotation vorticity field obtained using TDM on instantaneous large scale vector fields shown in Figure 9 at 14a: TV1 $(x=-100 \mathrm{~mm})$, 14b: TV2 $(x=-50 \mathrm{~mm}) \& 14 \mathrm{c}:$ TV3 $(x=75 \mathrm{~mm})$.

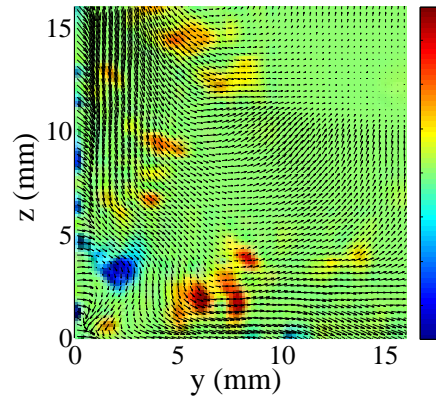

a)

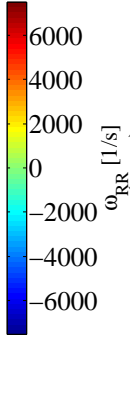

4000

000 ఏ

$000^{3} \mathrm{~N}$

000

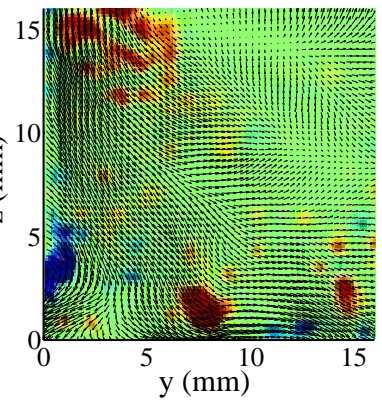

b)

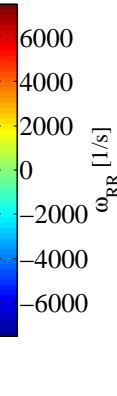

00

000 莡白 $000^{3^{\frac{\alpha}{\alpha}} \mathrm{E}}$

4000

000

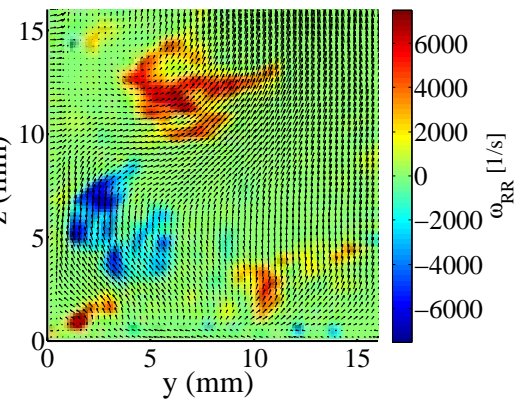

c)

Figure 15. Rigid rotation vorticity field of mean vector fields shown in Figure 5 at $15 \mathrm{a}:$ TV1 $(\boldsymbol{x}=-100 \mathrm{~mm}), 15 \mathrm{~b}:$ TV2 $(x=-50 \mathrm{~mm}) \&$ 15c: TV3 $(x=75 \mathrm{~mm})$. 


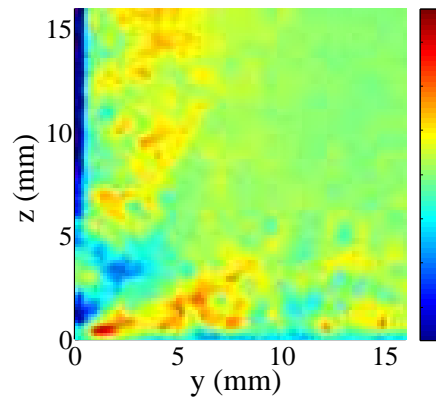

a)

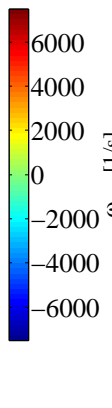

$000 \approx \overbrace{}^{10}$

$0 \quad \Xi \Xi$

5

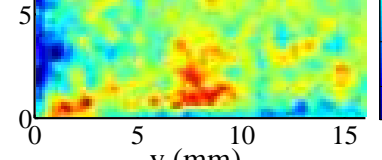

b)

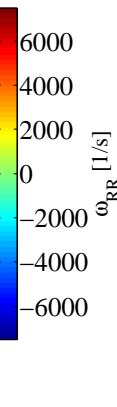

6000

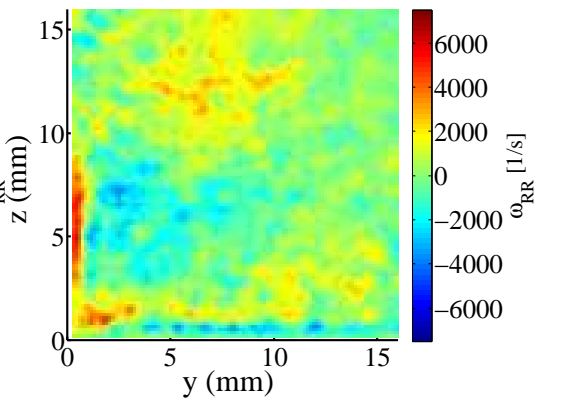

c)

Figure 16. Ensemble average of instantaneous rigid rotation vorticity fields at 16a: TV1 $(x=-100 \mathrm{~mm}), 16 \mathrm{~b}:$ TV2 $(x=-50 \mathrm{~mm}) \& 16 \mathrm{c}:$ TV3 $(x=75 \mathrm{~mm})$.

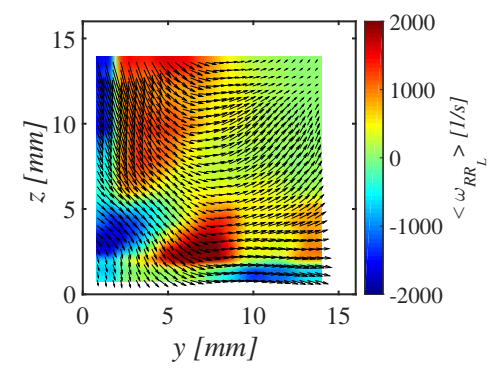

a)

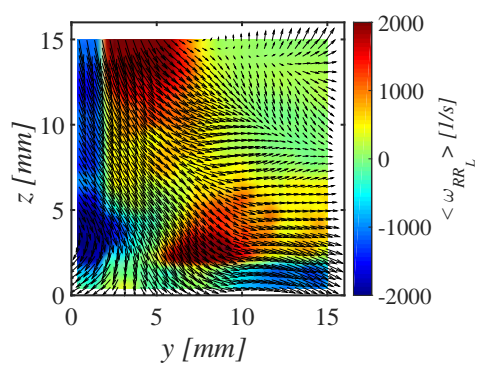

b)

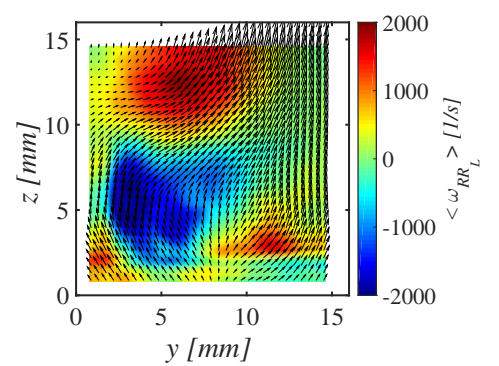

c)

Figure 17. Ensemble average of instantaneous rigid rotation vorticity fields obtained from large scale instantaneous vector fields at 17a: TV1 $(x=-100 \mathrm{~mm}), 17 \mathrm{~b}:$ TV2 $(x=-50 \mathrm{~mm}) \&$ 17c: TV3 $(x=75 \mathrm{~mm})$. 


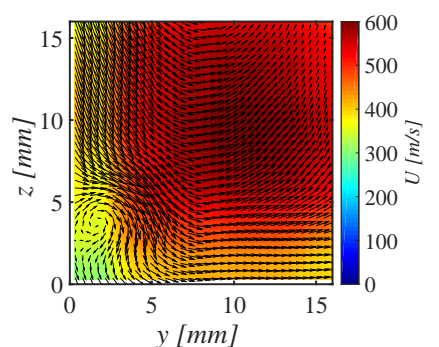

a)

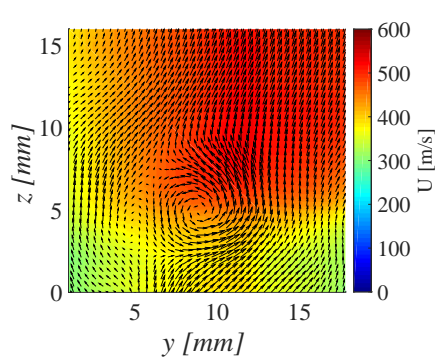

d)

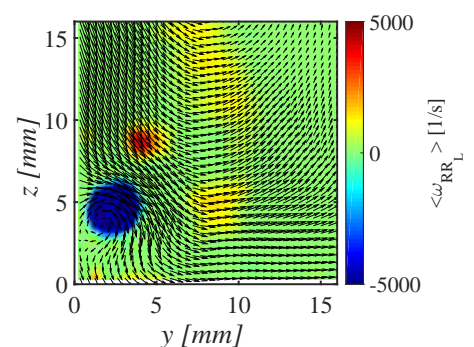

b)

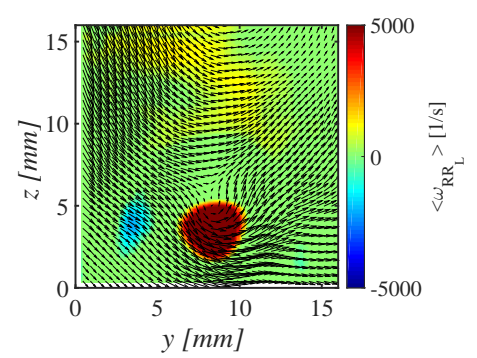

e)

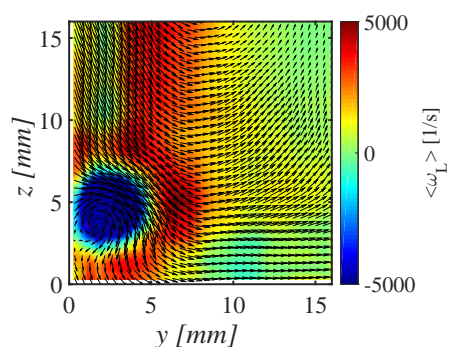

c)

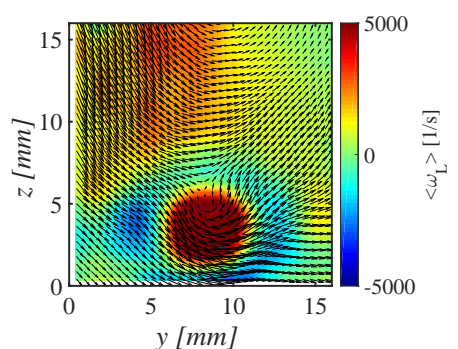

f)

Figure 18. 18a: Mean flow field obtained by conditional averaging of large scale flow field at TV1 by shifting origin to negative corner vortex center, 18b:TDM of 18a, 18c: Mean vorticity of 18a, 18d: Mean flow field obtained by conditional averaging of flow field by shifting origin to positive corner vortex center, 18e:TDM of 18d, 18f: Mean vorticity of $18 \mathrm{~d}$. 


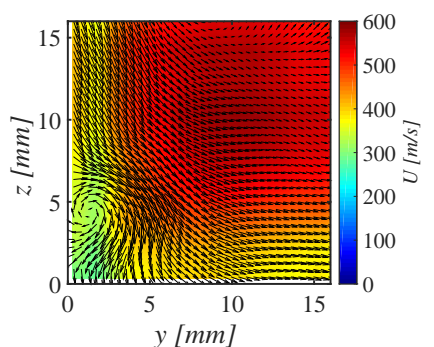

a)

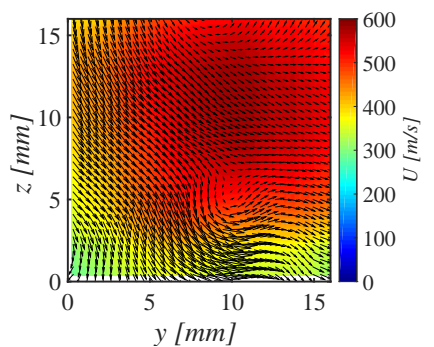

d)

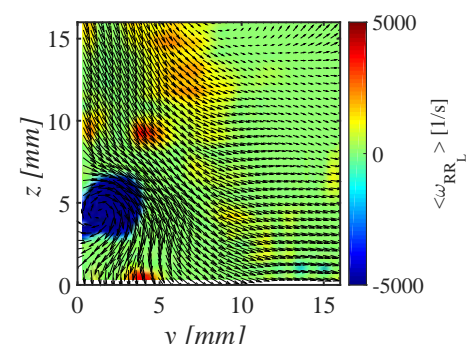

b)

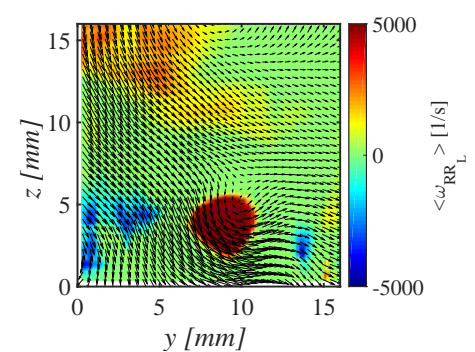

e)

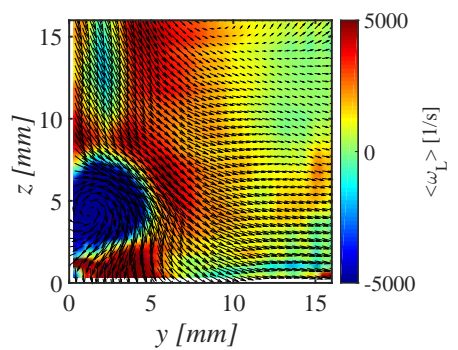

c)

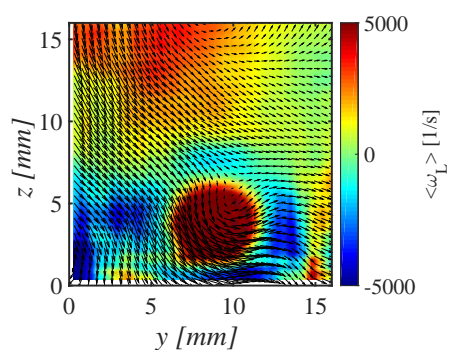

f)

Figure 19. 19a: Mean flow field obtained by conditional averaging of large scale flow field at TV2 by shifting origin to negative corner vortex center, 19b:TDM of 19a, 19c: Mean vorticity of 19a, 19d: Mean flow field obtained by conditional averaging of flow field by shifting origin to positive corner vortex center, 19e:TDM of 19d, 19f: Mean vorticity of 19d. 


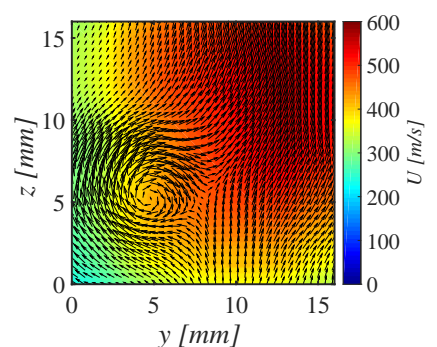

a)

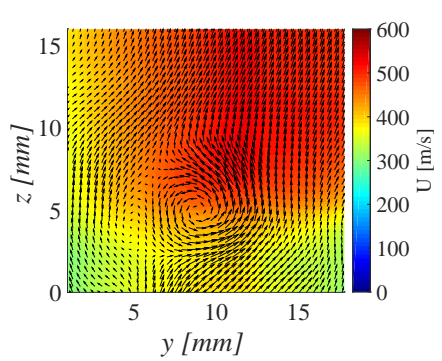

d)

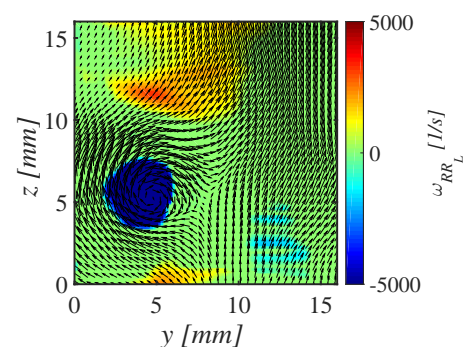

b)

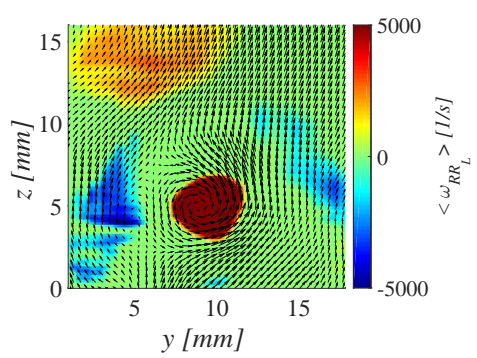

e)

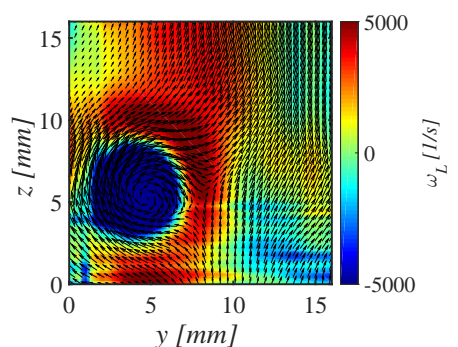

c)

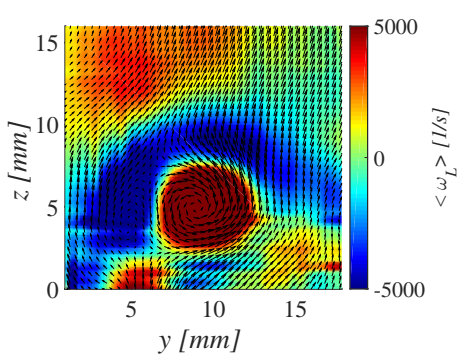

f)

Figure 20. 20a: Mean flow field obtained by conditional averaging of large scale flow field at TV3 by shifting origin to negative corner vortex center, 20b:TDM of 20a, 20c: Mean vorticity of 20a, 20d: Mean flow field obtained by conditional averaging of flow field by shifting origin to positive corner vortex center, 20e:TDM of 20d, 20f: Mean vorticity of $20 \mathrm{~d}$. 


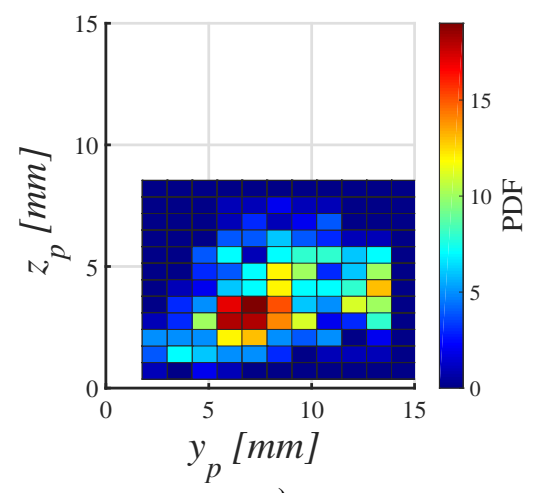

a)

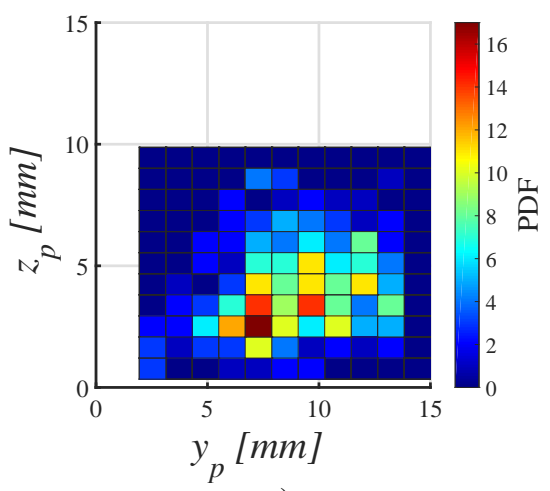

c)

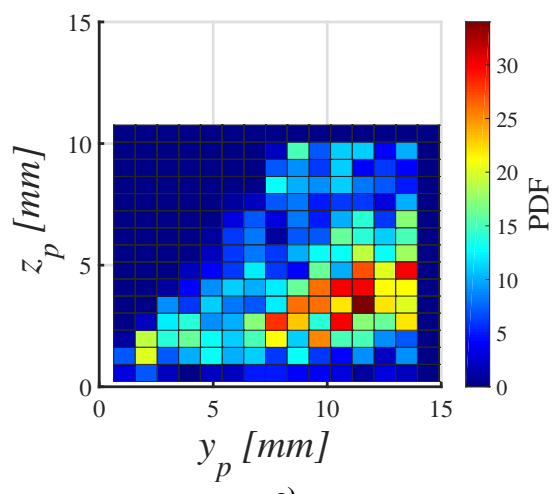

e)

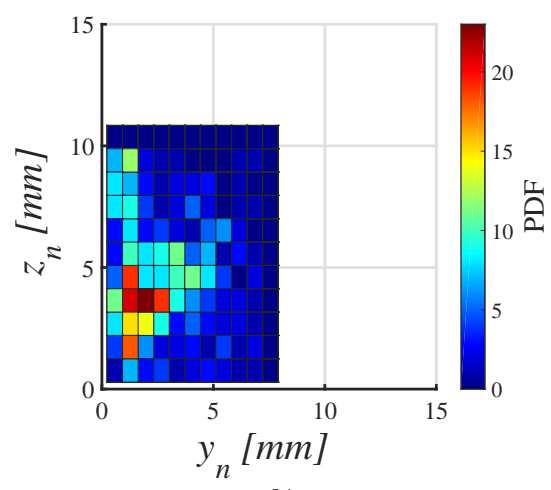

b)

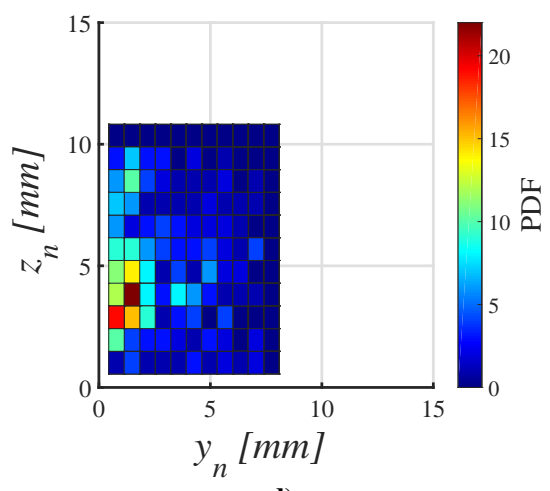

d)

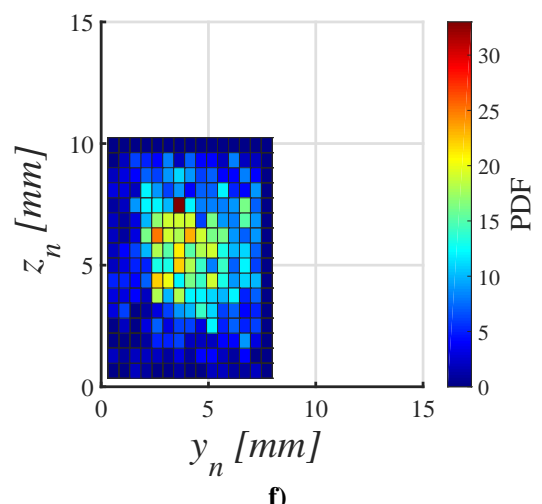

Figure 21. PDF of locations of corner vortex centers; 21a: Positive corner vortex center location at TV1, 21b: Negative corner vortex center location at TV1, 21c: Positive corner vortex center location at TV2, 21d: Negative corner vortex center location at TV2, 21e: Positive corner vortex center location at TV3 \& 21f: Negative corner vortex center location at TV3. 


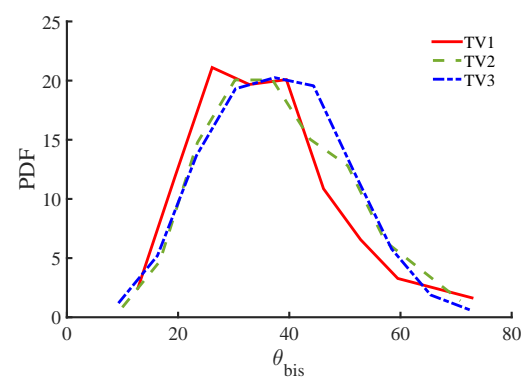

a)

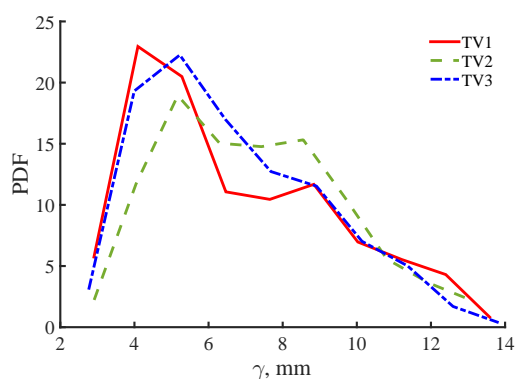

b)

Figure 22. Probability density functions of 22a: Corner bisector angle \& 22b: Corner vortex separation distance. 


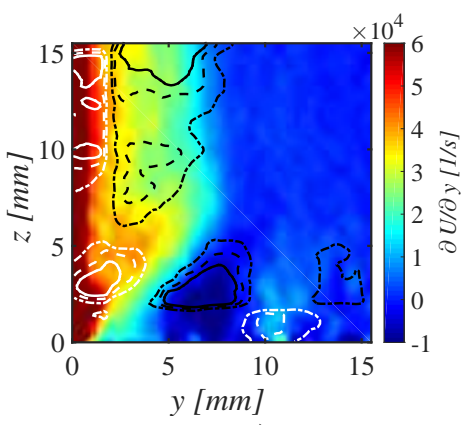

a)

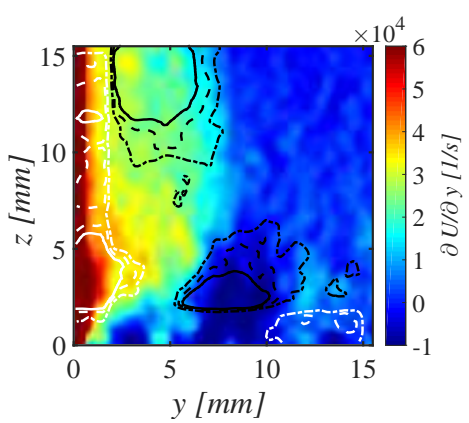

d)

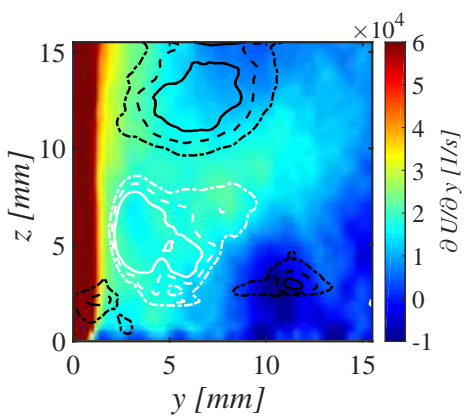

g)

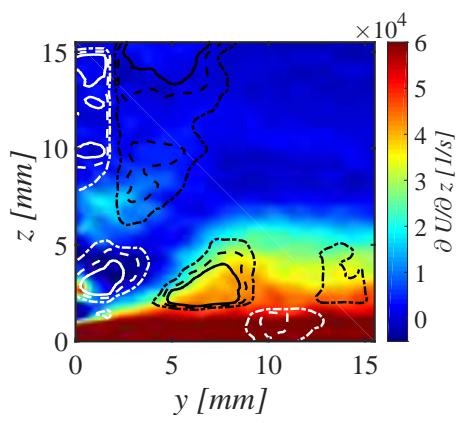

b)

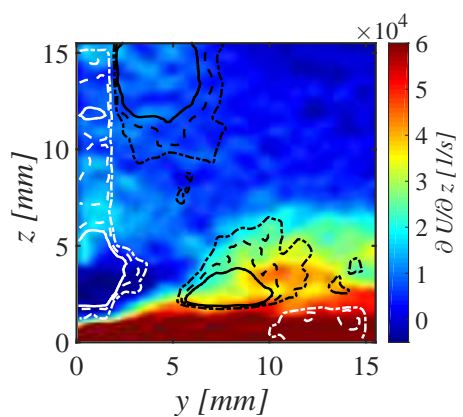

e)

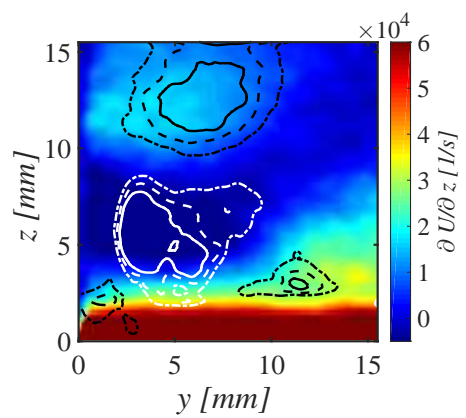

h)

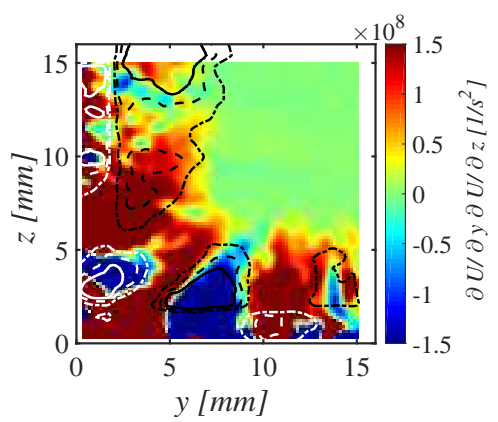

c)

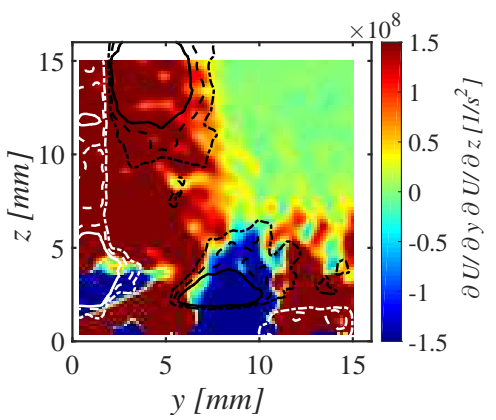

f)

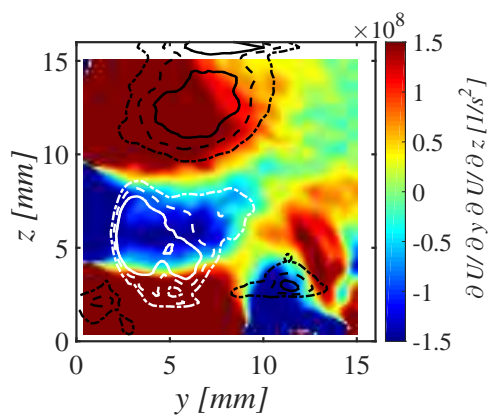

i)

Figure 23. Mean streamwise velocity in plane gradients and the product of the velocity gradients superimposed with contour lines of mean rigid rotation vorticity at large scales, white:negative vorticity, black: positive vorticity at 23a, 23b \& 23c: TV1; 23d, 23e \& $23 \mathrm{f}:$ TV2 \& 23g, 23h \& 23i: TV3. 


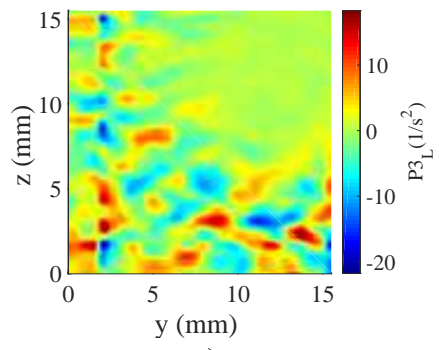

a)

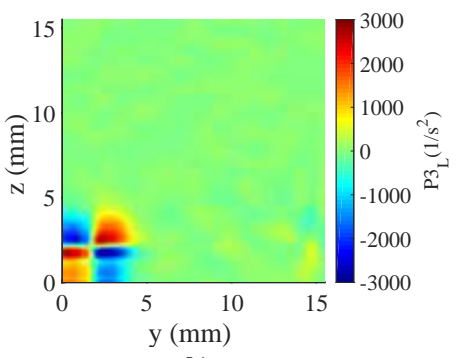

b)

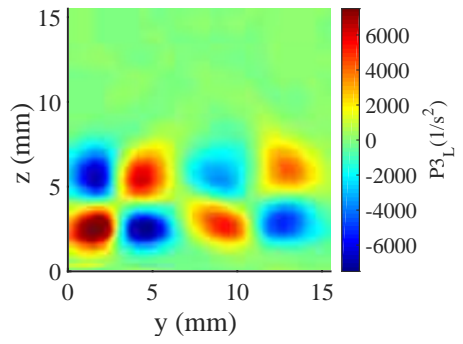

c)

Figure 24. Large scale vorticity production $P 3_{L}$ fields at 24a: TV1, 24b: TV2 and 24c: TV3.

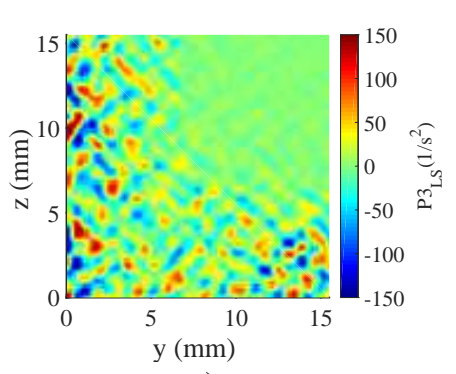

a)

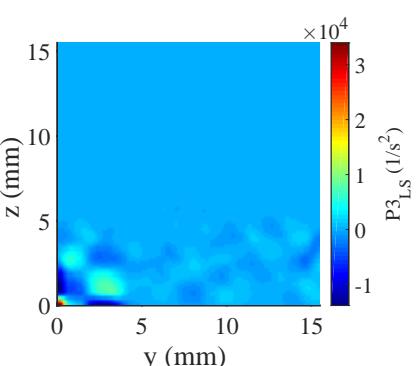

b)

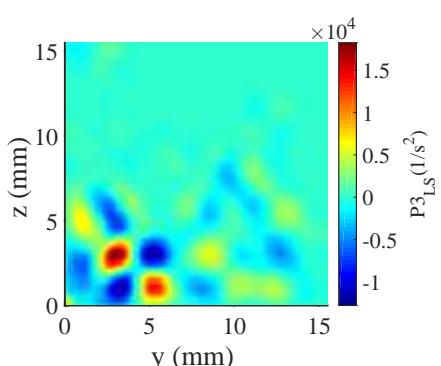

c)

Figure 25. Interscale vorticity production $P 3_{L S}$ fields at 25a: TV1, 25b: TV2 and 25c: TV3.

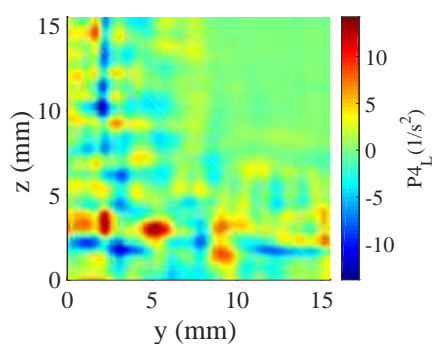

a)

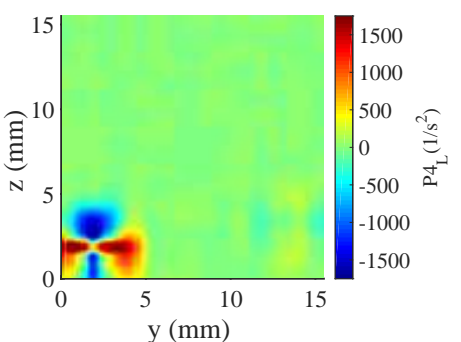

b)

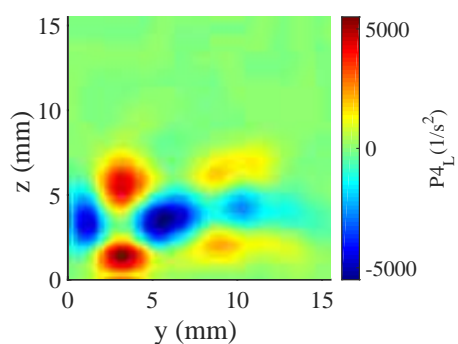

c)

Figure 26. Large scale vorticity production $P 4_{L}$ fields at 26a: TV1, 26b: TV2 and 26c: TV3.

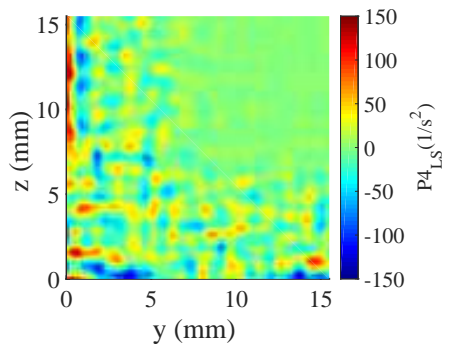

a)

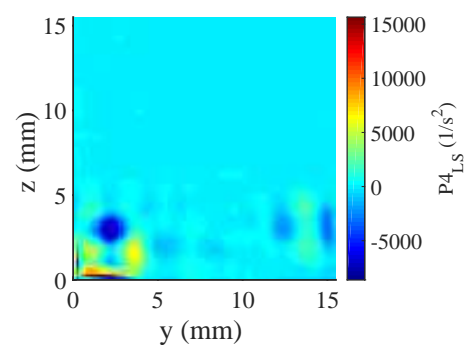

b)

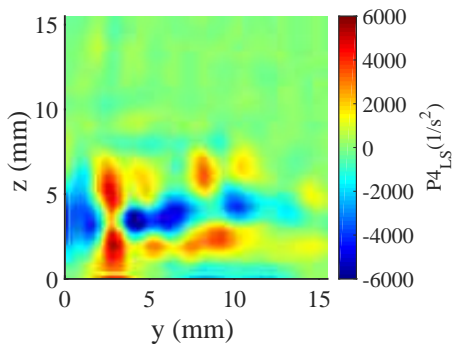

c)

Figure 27. Interscale vorticity production $P 4_{L S}$ fields at 27a: TV1, 27b: TV2 and 27c: TV3. 